\title{
Testing Thermal Gradient Driving Force for Grain Boundary Migration using Molecular Dynamics Simulations
}

\author{
Xian-Ming Bai,* Yongfeng Zhang, Michael R. Tonks \\ Fuels Modeling and Simulation Department, Idaho National Laboratory, Idaho Falls, ID 83415 \\ *Corresponding author. Email: Xianming.Bai@inl.gov
}

\begin{abstract}
Strong thermal gradients in low-thermal-conductivity ceramics may drive extended defects, such as grain boundaries and voids, to migrate in preferential directions. In this work, molecular dynamics simulations are conducted to study thermal gradient driven grain boundary migration and to verify a previously proposed thermal gradient driving force equation, using uranium dioxide as a model system. It is found that a thermal gradient drives grain boundaries to migrate up the gradient and the migration velocity increases under a constant gradient owing to the increase in mobility with temperature. Different grain boundaries migrate at very different rates due to their different intrinsic mobilities. The extracted mobilities from the thermal gradient driven simulations are compared with those calculated from two other well-established methods and good agreement between the three different methods is found, demonstrating that the theoretical equation of the thermal gradient driving force is valid, although a correction of one input parameter should be made. The discrepancy in the grain boundary mobilities between modeling and experiments is also discussed.
\end{abstract}

Keywords: Grain boundary migration; Thermal gradient driving force; Molecular dynamics; Oxides 


\section{Introduction}

Microstructural features in materials, such as grain boundaries (GBs), strongly affect the material properties such as mechanical strength [1], radiation tolerance [2], thermal conductivity [3], and electrical conductivity [4]. The migration of GBs causes grain growth and eventually changes the macroscopic properties of materials [5]. Therefore, understanding GB migration is critical for predicting the microstructural evolution in materials.

Many driving forces can drive GBs to migrate, such as curvature, stress, chemical driving force, magnetic fields, and thermal gradients [5]. Many of these driving forces have been systematically studied in the past [6-11]. However, the thermal gradient driving force has received less attention than others. Typically in metals the thermal gradient is not strong because they usually have high thermal conductivities. However, in some low-thermal-conductivity materials very large thermal gradients can build up. For example, uranium dioxides $\left(\mathrm{UO}_{2}\right)$ or mixed oxide (MOX) fuels are widely used as nuclear fuels in reactors. These materials have very low thermal conductivities, typically less than $10 \mathrm{~W} / \mathrm{m} \cdot \mathrm{K}$ at room temperature before irradiation [12], in contrast to $400 \mathrm{~W} / \mathrm{m} \cdot \mathrm{K}$ in copper [13]. After irradiation, the thermal conductivity of $\mathrm{UO}_{2}$ drops to about $2 \mathrm{~W} / \mathrm{m} \cdot \mathrm{K}$ [14]. As a result, the heat conduction from the hot center to the coolantcooled rim region in fuel rods is very low and a strong thermal gradient builds up during reactor operation. For example, the temperature drops about $1200{ }^{\circ} \mathrm{C} \sim 1600{ }^{\circ} \mathrm{C}$ under fast reactor conditions from the fuel center to rim region over about $0.5 \mathrm{~cm}$ [15]. Under such a large thermal gradient, some extended defects such as voids can migrate and cause the fuel restructuring. For example, in MOX fuels a central void can form due to the void migration driven by the thermal gradient [15]. GBs are another type of extended defects. Similar to voids, they may also migrate under a thermal gradient. Therefore, understanding how a thermal gradient drives GBs to migrate may help us understand whether this type of driving force causes a significant contribution to the complex fuel restructuring behavior in reactors.

Grain growth rate strongly correlates with the GB migration velocity. In general, when the driving force is not very strong, GB migration velocity $(v)$ is a product of the GB mobility $(M)$ and the driving force $F$ (actually in units of pressure) [5],

$$
v=M \times F .
$$

GB mobility is an intrinsic property of a GB and it can be described by a thermally activated relation with temperature $(T)[5]$,

$$
M=M_{0} \exp \left(\frac{-Q}{k_{B} T}\right),
$$

where $M_{0}$ is the prefactor of GB mobility, $Q$ is the activation energy for GB migration, and $k_{B}$ is the Boltzmann constant. Since the driving force for GB migration is the gradient of total free 
energy of the system, different driving mechanisms result in different theoretical formulations for the driving force. In the book by Gottstein and Shvindlerman [5], the thermal gradient driving force is expressed as:

$$
F=\frac{\Delta S \cdot 2 \lambda}{\Omega_{a}} \cdot \frac{d T}{d z},
$$

where $\Delta S$ is the entropy difference between a GB and a single crystal, $2 \lambda$ is GB thickness, $\Omega_{a}$ is material molar volume, $d T / d z$ is thermal gradient. However, no references or theoretical derivations are provided to explain the origin of this equation in the book. Here we attempt to derive this driving force equation from basic physical principles.

If a GB migrates along a thermal gradient, the only change in the Gibbs free energy of the entire system is the change of GB free energy with temperature. When the GB migrates from $z_{1}$ to $z_{2}$, the temperature changes from $T_{1}$ to $T_{2}$. The molar free energy of GB atoms (in units of $\mathrm{J} / \mathrm{mol}$ ) at position $z_{1}$ is:

$$
G_{1}=\Delta U_{1}-T_{1} \Delta S_{1}
$$

Similarly, the molar free energy of GB atoms at position $\mathrm{z}_{2}$ is:

$$
G_{2}=\Delta U_{2}-T_{2} \Delta S_{2}
$$

Here $\Delta U_{i}$ and $\Delta S_{i}(i=1,2)$ are the internal energy difference and entropy difference between GB atoms and bulk atoms in molar units, respectively. If the GB area is $A$, the GB region contains $N=\frac{A \cdot 2 \lambda}{\Omega_{a}}$ moles. If one assumes $\Delta U_{i}$ and $\Delta S_{i}$ are temperature-independent, i.e., $\Delta U_{1}=\Delta U_{2}=$ $\Delta U$ and $\Delta S_{1}=\Delta S_{2}=\Delta S$, the change of GB free energy in the entire system when a GB migrates from $z_{1}$ to $z_{2}$ is:

$$
d G=\left(G_{2}-G_{1}\right) \cdot N=\left(T_{1}-T_{2}\right) \Delta S \cdot N
$$

Since the driving force for GB migration is a per area quantity (pressure unit), $F$ is obtained by the energy gradient dividing by the area,

$$
F=-\frac{\partial G}{\partial z} \cdot \frac{1}{A}=-\frac{d G}{d z} \cdot \frac{1}{A}
$$

Using Eqs. (6) and (7), $d z=\left(z_{2}-z_{1}\right)$, and $N=\frac{A \cdot 2 \lambda}{\Omega_{a}}$, we obtain the expression of Eq. (3), 


$$
F=\frac{\Delta S \cdot 2 \lambda}{\Omega_{a}} \cdot \frac{T_{2}-T_{1}}{z_{2}-z_{1}}=\frac{\Delta S \cdot 2 \lambda}{\Omega_{a}} \cdot \frac{d T}{d z} .
$$

Note that validity of the assumptions made in this derivation (that $\Delta \mathrm{U}_{\mathrm{i}}$ and $\Delta \mathrm{S}_{\mathrm{i}}$ are temperatureindependent) is unclear. Therefore, this driving force equation should be tested before we use it.

In this work, we use molecular dynamics (MD) simulations to test the validity of the thermal gradient driving force equation, using $\mathrm{UO}_{2}$ as a model system. The testing strategy is briefly described as follows. First, the migration behavior of flat GBs under a strong thermal gradient is investigated. From these simulations, the GB migration velocity can be directly extracted and the thermal gradient driving force can be estimated using Eq. (3). Once the GB migration velocity and driving force are known, the GB mobility can be obtained using Eq. (1). Since GB mobility is an intrinsic property of each GB [16], different calculation methods using different driving forces should give similar GB mobilities. Therefore, if the GB mobility extracted from the thermal gradient driven simulations agrees well with those calculated from other standard methods, then Eq. (3) is the right theoretical equation to describe the thermal gradient driving force. To ensure the results are robust and not specific to one GB, multiple GBs of different GB characters are investigated in this work.

\section{Methods}

The MD simulation package LAMMPS [17] is used to study the GB motion driven by a thermal gradient in $\mathrm{UO}_{2}$. The widely used Basak potential [18] is used to describe the interaction between ions. This potential consists of a Coulomb, a Buckingham, and a Morse term. The parameters for these terms can be found elsewhere (noting that the units of some parameters in the original paper have typos) $[19,20]$. Previously, this potential has been successfully used to study radiation damage [19], defect cluster structures [20], GB structures [21], defect cluster migration [22], thermal conductivity [23], fracture [24, 25], void migration [26], and stress-induced phase transformation [24, 27] in $\mathrm{UO}_{2}$. In our simulations, the cutoff distance for the real-space Coulomb, Buckingham, and Morse terms is $11 \AA$. For the reciprocal-space summation of the Coulomb term, the particle-particle particle-mesh (PPPM) method [28] is used.

To exclude curvature driving force on GB motion and focus on thermal gradient driving force alone, five flat coincidence site lattice (CSL, or $\Sigma$ ) $\mathrm{GBs}$ in $\mathrm{UO}_{2}$ are studied: $\langle 110>\Sigma 3\{111\}$ coherent twin, $<100>\Sigma 5\{310\}$ symmetric tilt, $<100>\Sigma 5\{310\}$ twist, $<100>\Sigma 29\{520\}$ symmetric tilt, and $\langle 100\rangle \Sigma 25\{710\}$ symmetric tilt. Here they are referred to as $\Sigma 3$ twin, $\Sigma 5$ tilt, $\Sigma 5$ twist, $\Sigma 29$ tilt, and $\Sigma 25$ tilt, respectively. Their sizes and GB characters are shown in Table I. To some extent, these GBs represent a range of typical GB structures found in materials. For example, $\Sigma 3$ twin GB is usually a low-energy boundary; $\Sigma 5$ tilt GB has a relatively high frequency of presence among the special GBs as determined experimentally in $\mathrm{UO}_{2}$ [29]; $\Sigma 5$ twist GB represents high-angle twist boundaries; $\Sigma 29$ tilt GB is usually considered as a boundary close to 
random GBs; $\Sigma 25$ tilt GB has a misorientation angle of $16.3^{\circ}$ around $\langle 100\rangle$, which is a transition boundary between low- and high-angle boundaries. The initially unrelaxed GB structures are created using the online software GBStudio [30]. The gamma surface of each GB at $0 \mathrm{~K}$, which is the GB energy landscape with respect to the relative translations between two grains [31], is mapped out using molecular statics calculations. The minimum-energy GB structure in the gamma surface is used for studying GB motion. Since periodic boundary conditions are employed in all directions, there are two equivalent GBs in each simulation system.

In all simulations, a thermal gradient is imposed in the $\mathrm{z}$ direction, which has the longest length in each simulation system. The simulation setup is shown in Fig. 1(a). The hottest location is at the center, and the coldest locations are at both the top and bottom in the $\mathrm{z}$ direction. The two GBs are at $0.25 \times \mathrm{L}_{\mathrm{z}}$ and $0.75 \times \mathrm{L}_{\mathrm{z}}$ respectively, where $\mathrm{L}_{\mathrm{z}}$ is the length in the $\mathrm{z}$ direction for each GB-containing system. Since periodic boundary conditions are employed, the top and bottom regions in the $\mathrm{z}$ direction are actually the same cold location. The thickness of thermostated region in the $\mathrm{z}$ direction is $10 \AA$ at the hot center, and $5 \AA$ at each portion of the cold end (so the total thickness is also $10 \AA$ at the cold end). Unless mentioned otherwise, the coldest temperature is kept at $2300 \mathrm{~K}$ and the hottest at $3300 \mathrm{~K}$. Note that the melting temperature (solid-liquid coexistence temperature) of $\mathrm{UO}_{2}$ predicted by the Basak potential is about $3540 \mathrm{~K}$ [32], slightly higher than the experimental value of $3147 \mathrm{~K}$ [33]. Therefore the structure of the material at the hottest location still remains crystalline in our simulations. In all simulations, the time step is $2 \mathrm{fs}$. Before the simulation starts, the system volume is expanded to the equilibrium volume corresponding to the average temperature of the entire system, $2800 \mathrm{~K}$ in most cases. Initially, the entire system is relaxed at this temperature for 30,000 steps ( $0.06 \mathrm{~ns}$ ) in a constant-volume and constant-temperature (NVT) ensemble using the Nose-Hoover thermostat $[34,35]$. Then the simulation is performed in a constant-volume and constant-energy (NVE) ensemble and only the hot center and cold end (each is about $10 \AA$ in thickness) are thermostated by the velocity rescaling method [36] to maintain their respective temperatures efficiently. Note that under a thermal gradient, the center of mass of the entire system can have an artificial drift in the gradient direction [26, 37]. Therefore the velocity of each atom is adjusted by subtracting the center-of-mass velocity at each time step to neglect the drift of the entire system.

Using this setup, two equivalent temperature gradients build up due to the applied periodic boundary conditions. An example of the temperature profile along the $\mathrm{z}$ direction is shown in Fig. 1(b). The temperature difference between the hot center and cold end is about $1000 \mathrm{~K}$. Since the half-length in the $\mathrm{z}$ direction is about $100 \AA$ in all simulation systems, the thermal gradient is typically about $100 \mathrm{~K} / \mathrm{nm}$, nearly 6 orders of magnitude higher than those found in nuclear fuels. However, this high thermal gradient is necessary for observing GB motion at the MD simulation timescale.

To calculate the GB migration velocities, an accurate method to track GB positions at different times is needed. In this work, the GB positions were tracked using the uranium sub-lattice with 
the orientation parameter analysis method [38]. This parameter describes how the orientations between an atom and its nearest neighbors rotate with respect to a global reference orientation. Thus, it is a constant for atoms in the same grain, and varies with the grain orientation. The orientation parameter for the $i$ th atom $\left(f_{i}\right)$ is defined as [38]:

$$
f_{i}=\frac{1}{n} \sum_{j=1}^{n}\left(3-2 \sin ^{2} \theta_{i j}\right)^{2} \sin ^{2} \theta_{i j}
$$

where $n$ is the number of nearest neighbors of atom $i$ within a cutoff distance of $0.866 \times \mathrm{a}_{0}$, where $\mathrm{a}_{0}=5.6 \AA$ is the lattice parameter of the uranium sublattice; $j$ is the $j$ th neighboring atom of atom $i$ and $\theta_{i j}$ is the angle between a reference line vector and the line vector from atom $i$ and atom $j$. Note the coefficients in Eq. (9) may be adjusted to get a better distinction between two grains. Here we use the $\left[\begin{array}{lll}1 & 0 & 0\end{array}\right]$ direction of the top grain as the reference vector, and the average of the orientation parameters of the top and bottom grains as the cutoff. Figure 2 shows the comparison between the direct visualization of GB positions (Fig. 2(a)) and the characterized GB positions by the orientation parameter analysis (Fig. 2(b)). It is clear that this method accurately determines the GB positions. Note that a few atoms in the grain 2 have orientation parameters similar as in the grain 1 (and vice versa) due to the fluctuation noise at high temperatures. However, the number of such atoms is very small so that they are negligible for determining the GB positions.

Using this method, the number of uranium atoms in grain $1\left(N_{l}\right)$ or in grain $2\left(N_{2}\right)$ can be obtained as a function of time. If one assumes that the two GBs move the same distance from their initial positions, the time-dependent height of the lower portion of grain $1(l)$ shown in Fig. 2(b) can be estimated as,

$$
l=\frac{N_{1}}{N_{0}} \cdot \frac{L_{z}}{2}
$$

where $N_{0}\left(=N_{l}+N_{2}\right)$ is the total number of uranium atoms in the entire system, and $L_{z}$ is the length of the simulation system in the GB migration direction ( $\mathrm{z}$ direction). The average velocity of the two GBs is the time derivative of $l$,

$$
v=\frac{d}{d t} l=\frac{d}{d t} N_{1} \cdot \frac{L_{z}}{2 N_{0}} .
$$

Note that the GB local temperature keeps changing as GBs migrate under a thermal gradient. Since GB mobility is an exponential function of temperature (Eq. (2)), the GB velocity also keeps changing as GBs migrate according to Eq. (1). The GB local temperature at a given GB position $l$ is 


$$
T_{G B}=T_{l o w}+\frac{d T}{d z} \cdot l,
$$

where $T_{\text {low }}$ is the temperature at the cold end. If $T_{\text {high }}$ is the temperature at the hot center, the thermal gradient is

$$
\frac{d T}{d z}=\frac{\left(T_{\text {high }}-T_{\text {low }}\right)}{L_{z} / 2},
$$

Combining Eqs. (10), (12), and (13), the GB local temperature is also related to the number of uranium atoms in grain 1 :

$$
T_{G B}=T_{\text {low }}+\frac{\left(T_{\text {high }}-T_{\text {low }}\right) \cdot N_{1}}{N_{0}} .
$$

Therefore, if the number of uranium atoms in grain $1\left(N_{l}\right)$ at different simulation times is obtained, the GB migration velocity (v) can be estimated using Eq. (11) and the instantaneous GB local temperature can be estimated using Eq. (14).

\section{Results}

Firstly, the GB migration behavior is studied for five GBs. The temperature is $T_{\text {low }}=2300 \mathrm{~K}$ at the top/bottom and $\mathrm{T}_{\text {high }}=3300 \mathrm{~K}$ at the center. Four representative snapshots during the migration of the $\Sigma 5$ tilt GB are shown in Fig. 3. Initially the two GBs are in the middle between the cold end and hot center (Fig. 3(a)). Under the thermal gradient, the two GBs start to migrate towards the hot end. After the first time period of $0.8 \mathrm{~ns}$ (Fig. 3(b)), each GB migrates only about $7 \AA$ from their initial positions. After the second time period of $0.8 \mathrm{~ns}$ (Fig. 3(c)), each GB migrates substantially, about 30 35 A from their respective previous positions in Fig. 3(b). The upper GB migrates a slightly longer distance than the lower GB. After another $0.2 \mathrm{~ns}$ (Fig. 3(d)), the two GBs meet and anneal each other at the hot center and the system becomes a single crystal. During this shorter time period, each GB migrates about $15 \AA$ from their respective previous positions in Fig. 3(c).

Figure 3 clearly shows that the GB migration distance is not a linear function of time. In other words, GB migration velocity is not a constant due to the change in GB local temperature. Since GB mobility is an exponential function of temperature (Eq. (2)), its value increases during the migration. As a result, the GBs accelerate as they approach the hot center. Note that in previous MD simulations of void migration under a thermal gradient [26], the authors assumed that the void migration has a constant velocity and used a linear fit of the void migration distance against time to obtain the void migration velocity. This assumption might be valid when the void 
migration distance is small. In the present work, the GB migration distance is large and using such an approximation may result in inaccurate description of the GB migration behavior.

Since the thermal gradient driving force increases linearly with the thermal gradient (Eq. (3)), it is natural to think about using different thermal gradients to study the GB migration. For most cases in this work, a thermal gradient of about $100 \mathrm{~K} / \mathrm{nm}\left(\mathrm{T}_{\text {low }}=2300 \mathrm{~K}\right.$ and $\left.\mathrm{T}_{\text {high }}=3300 \mathrm{~K}\right)$ is used, as shown in Fig. 1(a). The initial temperature at GBs, which is also the average system temperature, is $2800 \mathrm{~K}$. Since $\mathrm{T}_{\text {high }}=3300 \mathrm{~K}$ is already close to the melting temperature of 3540 $\mathrm{K}$ predicted by Basak potential [32], the only way to increase the thermal gradient is to lower $\mathrm{T}_{\text {low }}$. Using $\mathrm{T}_{\text {low }}=1300 \mathrm{~K}$ and $\mathrm{T}_{\text {high }}=3300 \mathrm{~K}$, the thermal gradient increases by a factor of 2 , about $200 \mathrm{~K} / \mathrm{nm}$. However, the temperature at the initial GB locations is only $2300 \mathrm{~K}$, at which the GB mobility is very low for the MD timescale. Therefore, the incubation time for observing discernible GB migration is very long. For example, we have applied this larger thermal gradient for the $\Sigma 5$ tilt GB. Even after 2 million time steps (4 ns), the migration of two GBs is still indiscernible. The long incubation time increases the computational cost significantly, especially for low-mobility GBs. On the other hand, if a smaller thermal gradient of about $20 \mathrm{~K} / \mathrm{nm}$ is used $\left(\mathrm{T}_{\text {low }}=2700 \mathrm{~K}\right.$ and $\mathrm{T}_{\text {high }}=2900 \mathrm{~K}$ ), the driving force is too small to drive the GBs to move with a desired behavior. For example, we found that sometimes the two GBs do not move at all even after very long simulation time due to the small driving force, or they migrate randomly rather than towards the hot center due to fluctuation noise. After some experimentation, the coldest and hottest temperatures shown in Fig. 1(a) are determined to be the optimal setup for all GBs in this work.

For the five GBs shown in Table I, the maximum simulation time is $4.7 \mathrm{~ns}$. Since the domain lengths in the z-direction are similar for each GB type, the thermal gradients are also similar. Therefore, the GB mobilities can be qualitatively compared by simply comparing their migration velocities. As described in the Method section, the orientation parameter analysis is used to estimate the number of atoms in grain 1 and thus track GB positions (Eq. (10)) at different times. Here the number of atoms in grain 1 is obtained for every 1000 steps (0.002 ns). Figure 4 shows the normalized number of uranium atoms in grain 1 as a function of time for the five GBs, where the normalization is relative to the total number of uranium atoms (i.e., $N_{l} / N_{0}$ ) in their respective simulation systems. When two GBs anneal, the system becomes a single crystal so that $N_{1} / N_{0}=$ 1. Clearly the $\Sigma 29$ tilt and $\Sigma 5$ tilt GBs migrate very fast and the former migrates slightly faster; the $\Sigma 5$ twist GB migrates moderately; the $\Sigma 25$ tilt GB migrates very slowly; the $\Sigma 3$ twin GB does not migrate at the MD timescale. This result shows that different GBs have very different mobilities. The mobility sequence for the five GBs is $M_{\Sigma 3 \text { twin }}<M_{\Sigma 25 \text { tilt }}<M_{\Sigma 5 \text { twist }}<M_{\Sigma 5 \text { tilt }}$ $\mathrm{M}_{\Sigma 29}$ tilt.

To allow for a comparison between GB mobility and character, a qualitative description of the GB mobility is shown in Table I. From this comparison, GB mobility does not appear to have a clear correlation with any other GB characteristic (GB energy, misorientation angle, tilt/twist, or 
$\Sigma$ ). For the two high-mobility GBs ( $\Sigma 5$ tilt and $\Sigma 29$ tilt), both have a high GB energy and a high misorientation angle. Therefore, one may speculate that high-energy, high-angle GBs have a high GB mobility. However, many more GBs need to be tested to validate this hypothesis and this topic is beyond the scope of this work.

GB migration involves diffusion of atoms. In previous experimental work on $\mathrm{CeO}_{2}$, which has a similar fluorite structure as $\mathrm{UO}_{2}$, the GB mobility is assumed to correlate with the in-plane selfdiffusion of the slower diffusing cerium [39]. In $\mathrm{UO}_{2}$, the slower diffusing species is uranium. On the other hand, some theoretical studies [40] showed that GB self-diffusion and GB migration are two distinct processes. To investigate whether GB mobility correlates with the selfdiffusion of uranium atoms at GBs, MD simulations are conducted to calculate the GB selfdiffusion coefficients of uranium atoms at different temperatures. In these calculations, the constant-pressure and constant-temperature (NPT) ensemble is used. Each system contains about 10,000 total atoms and the simulation time is about $20 \mathrm{~ns}$. At each temperature, the mean square displacements (MSD) of uranium atoms as a function of time in the entire system at different directions are calculated. Since there are no pre-existing defects in the grain interior, the contribution from bulk diffusion to MSD is negligible and GB self-diffusion mainly contributes to the calculated MSD. From the linear fit of MSD against time (averaged by five runs) and using the Einstein relation [36], the diffusion coefficient $(D)$ in each direction is calculated at a given temperature for each GB. The Arrhenius plots of the GB self-diffusion coefficients $(\ln (D)$ vs. $1 / T$ ) in different directions are shown in Fig. 5, in which the linear fitting gives the activation energy for each data set. Since the uranium self-diffusion at the $\Sigma 3$ twin GB is very small, this GB is not included in Fig. 5. In the two in-plane directions (Figs. 5(a) and 5(b)), the selfdiffusion coefficients of the $\Sigma 5$ twist GB are very scattered so that a reliable activation energy cannot be obtained for each direction. However, it is evident that in the low-temperature regime (high $1 / T$ ), the self-diffusion coefficients of the $\Sigma 5$ twist GB are substantially higher than other GBs. For the $\Sigma 25$ tilt GB, the self-diffusion is faster in the $\mathrm{X}$ direction (which is also the rotation axis) but slower in the $\mathrm{Y}$ direction than the $\Sigma 5$ tilt and $\Sigma 29$ tilt GBs. On the other hand, the activation energies of the $\Sigma 25$ tilt GB in the two in-plane directions are much smaller than those of the other two GBs. However, none of these properties can be correlated to the GB mobility sequence of $\mathrm{M}_{\Sigma 25 \text { tilt }}<\mathrm{M}_{\Sigma 5 \text { twist }}<\mathrm{M}_{\Sigma 5 \text { tilt }} \sim \mathrm{M}_{\Sigma 29}$ tilt shown in Fig. 4. In the direction normal to GBs (Fig. 5(c)), which is also the GB migration direction, the $\Sigma 25$ tilt GB has the highest selfdiffusion coefficients in most of the temperature range and smallest activation energy, while the $\Sigma 5$ twist GB has the lowest self-diffusion coefficients and largest activation energy. Again, these properties cannot be correlated with the mobility sequence shown in Fig. 4. Therefore, although the GB mobility might be related to the GB self-diffusion, the connection between them is not very straightforward. This conclusion is consistent with the study of the correlation between GB self-diffusion and GB migration in copper by Schönfelder et al [40]. 
Figure 4 only provides a qualitative comparison of GB mobility between different GBs. To quantitatively calculate the GB mobility, some additional analysis is conducted. Since neither $\Sigma 3$ twin nor $\Sigma 25$ tilt GBs significantly migrate, they are excluded from the quantitative study of GB mobility. According to Eq. (1), the GB migration velocity is needed for estimating GB mobility. According to Eq. (11), the GB migration velocity is related to the time-derivative of the number of atoms in grain 1. As mentioned earlier, the GB migration velocity keeps changing as it migrates under a thermal gradient because the mobility is a function of temperature. Therefore, one cannot simply use a linear fit of the data shown in Fig. 4 against time to extract the GB velocity for each GB. As an approximation, the number of atoms in grain 1 is fitted with a $2^{\text {nd }}$ order polynomial function of time for each GB. An example of the polynomial fitting for the $\Sigma 5$ tilt GB is shown in Fig. 6(a). The fitting gives

$$
\mathrm{N}_{1}=18922.8-940.6 \times \mathrm{t}+5002.3 \times \mathrm{t}^{2} \text {. }
$$

The time derivative of Eq. (15) gives

$$
\mathrm{dN}_{1} / \mathrm{dt}=-940.6+10004.6 \times \mathrm{t}
$$

The time-dependent GB velocity can be estimated by replacing $\mathrm{dN}_{1} / \mathrm{dt}$ in Eq. (11) with Eq. (16). At a time $t$, the GB local temperature can be calculated using the combination of Eqs. (14) and (15). Therefore, the temperature-dependent GB velocity also can be obtained. An example is shown in Fig. 6(b).

The thermal gradient driving force $(F)$ can be estimated using Eq. (3). Here for the entropy difference between a GB and a single crystal, we use the value of $\Delta S=8 \times 10^{3} \mathrm{~J} / \mathrm{mol} / \mathrm{K}$ provided in the book by Gottstein and Shvindlerman [5]. The GB thickness is assumed to be $2 \lambda=1 \mathrm{~nm}$, the molar volume of $\mathrm{UO}_{2}$ molecules is about $\Omega_{a}=2.44 \times 10^{-5} \mathrm{~m}^{3} / \mathrm{mol}$, and the thermal gradient in the simulation is $d T / d z=103.5 \mathrm{~K} / \mathrm{nm}$. The temperature-dependent GB mobility is calculated by using Eq. (1), $M=v / F$, where $v$ is the same as shown in Fig. 6(b). The Arrhenius plot of the calculated GB mobility for the $\Sigma 5$ tilt GB is shown in Fig. 7. Previously, Ainscough et al. [41] measured the GB mobility of $\mathrm{UO}_{2}$ using sintering experiments. They also collected some experimental data obtained by others. These experimental data are shown in Fig. 7 for comparison with our simulation results. In experiments, the temperature was in the range between $1300 \mathrm{~K}$ and $2440 \mathrm{~K}$, which are much lower than in MD simulations. If one assumes the experimental mobility follows an Arrhenius behavior (Eq. (2)), the experimental data can be linearly extrapolated to the high temperature regime in Fig. 7 and compared with our MD simulation results directly. Clearly, the extrapolated experimental data are only about 1 order of magnitude smaller than our calculated mobilities at high temperatures. Given the fact that our MD simulation is a completely independent study to the experiments, the agreement seems to be very good. 
However, the seemly excellent agreement between simulations and experiments in Fig. 7 is somewhat surprising. In simulations the materials are defect-free (except for GBs themselves) and impurity-free. Moreover, the $\Sigma 5$ tilt GB is a special CSL GB with an ideal GB structure. In reality, especially in sintering experiments, most GBs are not ideal. More importantly, the experimental samples contain many cavities (e.g., pores) and impurities. They can cause either void-pinning effects [42] or impurity-drag effects [43] to slow down the GB motion. For example, previous experiments showed that the GB mobility can be lowered by a few orders of magnitude if a small percentage of impurities are added to the materials [5]. Therefore, the effective mobility extracted from sintering experiments should be much lower than that calculated from MD simulations. Therefore, the good agreement between modeling and experiments in Fig. 7 could be a coincidence.

For thermal gradient driving force (Eq. (3)), two parameters - GB thickness (2 $\lambda$ ) and entropy difference between a single crystal and a GB $(\Delta S)$ - are not well defined. In previous experiments [44], researchers showed that GB thickness is about a few nanometers and close to the GB periodicity length. Therefore, the value of $2 \lambda=1 \mathrm{~nm}$ seems to be reasonable. The entropy difference $\Delta S$ may be very difficult to accurately estimate as the method for measuring or calculating it has not been well established to the best of our knowledge. However, if one assumes the extreme case of the GB structure being a liquid-like amorphous structure, the upper limit of this entropy term is the entropy difference between a crystalline solid and a liquid, or the entropy of melting, $\Delta S_{m}$. To calculate the entropy of melting, a $\mathrm{UO}_{2}$ single crystal consisting of 6144 atoms is heated from $1000 \mathrm{~K}$ to $5000 \mathrm{~K}$ to let it become a liquid. Then the liquid is cooled from $5000 \mathrm{~K}$ to $1000 \mathrm{~K}$. In this MD simulation, NPT ensemble is used with a zero external pressure. At every 10,000 steps $(0.02 \mathrm{~ns})$, the temperature is increased or decreased by $100 \mathrm{~K}$. So the heating/cooling rate is $5000 \mathrm{~K} / \mathrm{ns}$. The enthalpy per $\mathrm{UO}_{2}$ molecule at different temperatures during this heating-cooling process is recorded, as shown in Fig. 8. The enthalpy increases sharply at about $4300 \mathrm{~K}$, indicating the crystal becomes a liquid at this temperature. However, this transition temperature is the superheating limit of the crystal because the surface melting is suppressed by the employment of periodic boundary conditions in MD simulation [45]. The true melting temperature $\left(T_{m}\right)$ is the solid-liquid coexistence temperature, which is usually much lower than the superheating limit. Govers et al. [32] calculated the solid-liquid coexistence temperature predicted by Basak potential to be about $T_{m}=3540 \mathrm{~K}$. The enthalpy difference between the solid and liquid at this melting temperature is the latent heat of melting $\left(\Delta \mathrm{H}_{\mathrm{m}} \approx 0.57\right.$ $\mathrm{eV}$ ), as shown in Fig. 8. Thus the entropy of melting for $\mathrm{UO}_{2}$ is

$$
\Delta S_{m}=\frac{\Delta H_{m}}{T_{m}} \approx 15.5 \mathrm{~J} / \mathrm{mol} / \mathrm{K}
$$

Note that this entropy difference is the upper limit of the entropy difference between a GB and a crystal. Clearly, this value is about 3 orders of magnitude smaller than that provided in the book 
by Gottstein and Shvindlerman [5], $\Delta S=8 \times 10^{3} \mathrm{~J} / \mathrm{mol} / \mathrm{K}$. In metals, the entropy of melting is close to the ideal gas constant, $R=8.31 \mathrm{~J} / \mathrm{mol} / \mathrm{K}$ [46], a value close to our MD calculation. Therefore, the book must have a typo - the authors may have meant 8 rather than $8000 \mathrm{~J} / \mathrm{mol} / \mathrm{K}$. However, this typo makes the calculated GB mobility about 3 orders of magnitude lower than it should be in Fig. 7.

To further test the thermal gradient driving force, two well-established methods are used to calculate the GB mobility in MD simulations. Since GB mobility is an intrinsic property, different methods should give a similar value for the same GB [16], or at least the difference should not be more than a few orders of magnitude. The two methods are curvature-driven GB migration [9] and GB random walk [10]. Previously these methods have been applied to calculate GB mobilities in many materials, though primarily in pure metals [38, 47-50]. Here we use the two methods to calculate the GB mobility in a multi-component ionic system, $\mathrm{UO}_{2}$. The details of using these two methods for calculating $\mathrm{GB}$ mobility in $\mathrm{UO}_{2}$ will be described elsewhere and only a brief description is provided here.

In the first method, a circular grain is embedded in a matrix (Fig. 9(a)). The simulation system has a quasi-2D geometry so that the circular grain can be viewed as a disk. The GB created in this way can be viewed as a random GB. Therefore, this method cannot be used to study special CSL GBs. The dimension of the matrix is $282.4 \AA$ in the $\mathrm{x}$ and $\mathrm{y}$ directions and $22.6 \AA$ in the $\mathrm{z}$ direction. The initial radius of the circular grain is about $R=100 \AA$. The circular grain is rotated about $45^{\circ}$ along the normal direction of the circular grain ( $\mathrm{z}$ axis) from the matrix. To avoid overlapping of atoms at GBs, some GB atoms are removed so that the minimum interatomic distance between uranium atoms is $3.8 \AA$. The number of total atoms in the simulation system is 119,808 . The simulation system is then brought to a high temperature between $2500 \mathrm{~K}$ and 3000 $\mathrm{K}$ and the simulation is conducted in a NPT ensemble at zero external pressure. At these temperatures, the circular grain shrinks due to the curvature driving force. The shrink velocity of the disk radius is related to the GB mobility $(M)$, disk radius $(R)$, and the grain boundary energy

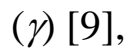

$$
v=\frac{d}{d t} R=M \cdot \frac{\gamma}{R} .
$$

Here the GB energy $\gamma=1.5 \mathrm{~J} / \mathrm{m}^{2}$ is used, which is close to that of the high-energy GBs shown in Table 1. Once the shrinking velocity is obtained from MD simulations, the mobility can be estimated using Eq. (18).

The second method (random walk method) can be used to study the mobility of special CSL GBs. In this method, a flat GB is brought to a high temperature between $2500 \mathrm{~K}$ and $3000 \mathrm{~K}$ and again the simulation is conducted in a NPT ensemble at zero external pressure. At these high temperatures, the central GB fluctuates around its initial position (Fig. 9(b)). The average GB 
fluctuation profile $<\bar{h}^{2}>$ is calculated to estimate GB mobility with the relation [10],

$$
<\bar{h}^{2}>=\frac{2 M k_{B} T}{A} t
$$

where $A$ is GB area and $t$ is the simulation time. Using this method, the mobilities are calculated for the same three CSL GBs ( $\Sigma 5$ tilt, $\Sigma 29$ tilt, $\Sigma 5$ twist) that were studied in the thermal gradient driven method. The simulation systems typically contain about 57,600 total atoms and the dimension is $22.6 \AA$ along the $\mathrm{x}$ direction, $178.4 \AA$ along the $\mathrm{y}$ direction (which is the rotation axis direction), and $215.2 \AA$ along the $\mathrm{z}$ direction (GB normal).

The comparison of the GB mobility obtained from three different methods is shown in Fig. 10(a). For the random-walk method, the calculated GB mobilities of the three CSL GBs have the

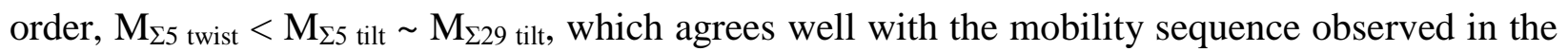
thermal gradient driven method (Fig. 4). For the curvature-driven method, the calculated mobilities are close to those of the two high-mobility GBs ( $\Sigma 5$ tilt and $\Sigma 29$ tilt) calculated by the random-walk method, suggesting that these two high-mobility GBs are similar to random GBs. For the thermal gradient driven method, mobilities are estimated using both the wrong entropy value provided in the book $\left(\Delta S=8 \times 10^{3} \mathrm{~J} / \mathrm{mol} / \mathrm{K}\right)$ and the entropy of melting value calculated from MD $\left(\Delta S_{m}=15.5 \mathrm{~J} / \mathrm{mol} / \mathrm{K}\right)$. Clearly, if the wrong $\Delta S$ is used, the estimated GB mobility is about 3 orders of magnitude lower than those calculated from the two standard methods. On the other hand, if the MD-calculated $\Delta S_{m}$ is used, the GB mobility agrees very well with those calculated from the two standard methods. Since the thermal gradient driving force is used as inputs for calculating GB mobility, the good agreement also proves that the thermal gradient driving force equation (Eq. (3)) is valid, as long as a reasonable value for $\Delta S$ is used in this equation. Note that the MD-calculated $\Delta S_{m}$ is the upper limit of $\Delta S$. If the true $\Delta S$ is half of $\Delta S_{m}$, the calculated mobilities from the thermal gradient driven method would only shift upwards slightly in the logarithm plot of Fig. 10(a) and the agreement between different methods would still be good.

The comparison between MD-calculated mobilities and experimental data is shown in Fig. 10(b), which is a combined plot of Fig. 7 and Fig. 10(a). Clearly, the discrepancy between modeling and experiment is very large - the former is about four orders of magnitude higher than the later. As discussed earlier, clean and ideal GBs are used to calculate the intrinsic mobility in modeling. But in reality, the GB mobility measured from sintering experiments is strongly affected by solute drag and void pinning effects. Therefore, the experimental mobility, which is dominated by extrinsic mobility, should be much lower than that obtained in modeling. Ironically, using the wrong entropy value the modeling provides much better agreement between modeling and experiment. This is a good example of the fact that a seemly good agreement between modeling and experiments does not necessary signify that the underlying physics used in the model is correct. 


\section{Discussion}

In reality, the thermal gradient driving force can coexist with many other driving forces, among which the curvature driving force is nearly always present. Therefore, an assessment of their relative importance is helpful to understanding how they contribute to grain growth. The curvature driving force is related to the GB energy $(\gamma)$ and grain size (or diameter, $D$ ) [5],

$$
F=\frac{2 \gamma}{D} .
$$

Here a standard value of $\gamma=1.5 \mathrm{~J} / \mathrm{m}^{2}$ is used for calculating the curvature driving force. For calculating the thermal gradient driving force (Eq. (3)), the typical thermal gradient in light water reactor (LWR) fuels is used, $d T / d z=0.2 \mathrm{~K} / \mu \mathrm{m}$. The GB thickness is still assumed to be $2 \lambda=1$ $\mathrm{nm}$ and the MD-calculated entropy value of $\Delta S=15.5 \mathrm{~J} / \mathrm{mol} / \mathrm{K}$ is used. The comparison of the two types of driving forces as a function of grain size is shown in Fig. 11. Since the curvature driving force is an inverse function of the grain size, it decreases linearly with grain size in the $\log -\log$ plot. The thermal gradient driving force is independent of grain size so it remains constant. Clearly, the curvature driving force is much larger than the thermal gradient driving force for a wide range of grain sizes. At the typical fuel grain size of $D=10 \mu \mathrm{m}$, the curvature driving force is still about 3 orders of magnitude higher than the thermal gradient driving force. By equating the two driving forces (Eqs. (3) and (20)), the critical grain size for the thermal gradient driving force being more dominant than curvature driving force is,

$$
D^{*}=\frac{2 \gamma \cdot \Omega_{a}}{\Delta S \cdot 2 \lambda} \cdot \frac{d z}{d T}=4.6 \mathrm{~cm},
$$

which is even larger than the typical fuel rod diameter of $1 \mathrm{~cm}$. This result suggests that the thermal gradient driving force is negligible in comparison with the curvature driving force in realistic conditions. In our previous phase field modeling of $\mathrm{UO}_{2}$ grain growth under a thermal gradient [51], the wrong entropy value specified in the book was directly used because at that time we had not performed MD simulations to check the validity of the thermal gradient driving force. Even so, the main conclusion from that work, that the thermal gradient driving force is not very important for grain growth in light water reactor conditions, is only strengthened if a reasonable entropy value is used (as shown in Fig. 11).

Although the thermal gradient driving force is not important for driving GB migration, the thermal gradient itself still has a large effect on grain growth behavior because of the sensitivity of the GB mobility to temperature (Eq. (2)). Although thermal gradient driving force is negligible, other driving forces such as curvature driving force can cause significantly more grain growth at the hot center than at the cold end due to the difference in GB mobility at different 
regions. Therefore, although the thermal gradient driving force can be ignored, other effects caused by the thermal gradient cannot be ignored.

This work also provides a new way to calculate the GB-character-dependent mobility using MD simulation. In the curvature-driven method, only an average GB mobility can be obtained. In the GB random-walk method, the GB-character-dependent mobility can be obtained for different GBs, but this method requires a very long simulation time to get statistically meaningful GB fluctuation profiles. In contrast, the mobilities of different special GBs can be calculated relatively straightforwardly using the thermal gradient driven method, as shown in Fig. 10(a). However, one drawback of this method is that the calculated mobility is only limited to a narrow range of high temperatures. If one extrapolates the high-temperature data to the low-temperature regime using Arrhenius relation, caution should be taken because the empirical polynomial fitting used for estimating GB velocity in Fig. 6(a) may induce some error. Such error can propagate and be magnified if the extrapolation range is large. In the future, if a more physically sound relation can be developed to fit the simulation data shown in Fig. 6(a), the error propagation could be suppressed. Moreover, an upper bound value is used for the $\Delta S$ in Eq. (3). In this work, we assumed that this entropy term is the same for all GBs. This assumption may induce some additional error in calculating the GB mobility. Nevertheless, this method still provides a fast and easy way to qualitatively evaluate how GB mobility depends on GB character, as shown in Fig. 4.

Under a large thermal gradient, a thermal stress gradient can build up. In our simulations, the system volume is fixed to the equilibrium volume for the average system temperature, $2800 \mathrm{~K}$. Therefore, the material tries to shrink at the cold end and to expand at the hot center. As a result, the cold end experiences a tensile stress while the hot center is under a compressive stress. Since stress also can be a driving force for GB migration [52], it is important to evaluate whether the thermal stress affects the GB migration. If thermal stress were driving GB migration, the stress should gradually decrease as the GBs migrate to lower the Gibbs free energy of the entire system. Figure 12 shows the in-plane stress profiles in the $\Sigma 5$ tilt GB system along the GB migration direction ( $\mathrm{z}$ direction) before the two GBs start to migrate at $0.14 \mathrm{~ns}$ and after the two GBs anneal at $2.4 \mathrm{~ns}$. The stress is the average stress of the two in-plane directions, $\left(\sigma_{\mathrm{xx}}+\sigma_{\mathrm{yy}}\right) / 2$. As expected, the hot center is under a compressive stress and the cold end is under a tensile stress. The stress gradient is about $0.4 \mathrm{GPa} / \mathrm{nm}$. Clearly, the thermal stress profiles before and after GB migration are nearly identical, indicating the migration of GBs does not release the stress. Actually, the magnitude of the compressive stress at the hot center increases slightly after the two GBs anneal, which is opposite of what would happen if the stress were driving GB migration [7]. Therefore, similar to the void migration under a thermal gradient [26], we conclude that thermal stress also has a negligible effect on the GB migration. 


\section{Conclusions}

In this work, the GB migration behavior driven by a thermal gradient in $\mathrm{UO}_{2}$ is investigated using MD simulations. Five representative GBs of different GB character are studied. The qualitative comparison shows that their mobilities follow the order: $M_{\Sigma 3 \text { twin }}<M_{\Sigma 25 \text { tilt }}<M_{\Sigma 5 \text { twist }}$

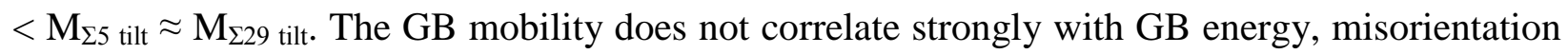
angle, tilt/twist, CSL $\Sigma$ number, or GB self-diffusion, suggesting that GB mobility is a complex material property and it is not a simple function of any single GB property.

The major goal of this work is to test the validity of the thermal gradient driving force equation proposed previously. Using the GB migration velocity obtained from MD simulations and the theoretical thermal gradient driving force equation, the mobilities of three GBs are calculated quantitatively. The obtained GB mobilities are compared with those calculated from two wellestablished methods and good agreement between the three methods is demonstrated. The good agreement proves that the previously proposed theoretical equation of the thermal gradient driving force is valid, however the entropy term that is suggested is about three orders of magnitude larger than it should be, possibly due to a typo in the original reference. Although this typo seems to be a trivial mistake, it can be easily missed. The validated theoretical equation of the thermal gradient driving force can then be used in mesoscale modeling such as phase field method [51] to study the GB migration behavior under a thermal gradient, if necessary.

Interestingly, the thermal gradient driving force is about three orders of magnitude smaller than the curvature driving force at the light water reactor fuel conditions. This result suggests that this driving force may be neglected for modeling GB motion in many practical applications. However, it does not mean that the thermal gradient can be neglected for grain growth. The thermal gradient induces significant difference in temperature (and thus GB mobility) along the temperature gradient in materials. Accordingly, other driving forces can drive GBs to migrate differently and result in different grain growth behavior at different locations.

\section{Acknowledgement}

This work is supported by the US Department of Energy, Office of Nuclear Energy, Nuclear Energy Advanced Modeling and Simulation (NEAMS) Program. The method of creating grain boundary structures of $\mathrm{UO}_{2}$ were developed under the support of The Center of Materials Science for Nuclear Fuels, an Energy Frontier Research Center funded by US Department of Energy, Office of Basic Energy Sciences (FWP\# 1356). This manuscript was authored by a contractor (Battelle Energy Alliance, LLC) of the US Government under the Department of Energy Contract No DE-AC07-05ID14517. Accordingly, the US Government retains and the publisher, by accepting the paper for publication, acknowledges that the US Government retains 
a nonexclusive, paid-up, irrevocable, world-wide license to publish or reproduce the published form of this manuscript, or allow others to do so, for US Government purposes. 
Table I. The sizes and GB characters of the five GBs studied in this work. The resulting qualitative comparison of GB motion from thermal gradient driven simulations is shown in the last row for comparison.

\begin{tabular}{|l|l|l|l|l|l|}
\hline GB type & $\Sigma 3\{111\}$ twin & $\Sigma 5\{310\}$ tilt & $\Sigma 5\{310\}$ twist & $\Sigma 29\{520\}$ tilt & $\Sigma 25\{710\}$ tilt \\
\hline $\begin{array}{l}\text { Number } \\
\text { of atoms }\end{array}$ & 120,960 & 110,160 & 108,000 & 116,280 & 102,816 \\
\hline $\begin{array}{l}\mathrm{L}_{\mathrm{x}} \times \mathrm{L}_{\mathrm{y}} \times \mathrm{L}_{\mathrm{z}} \\
\left(\AA^{3}\right)\end{array}$ & $97.0 \times 96.0 \times 196.0$ & $96.2 \times 89.5 \times 193.2$ & $89.5 \times 89.5 \times 203.7$ & $96.2 \times 91.4 \times 199.6$ & $96.2 \times 80.0 \times 201.7$ \\
\hline $\begin{array}{l}\text { Inclination } \\
\text { angle }\end{array}$ & $<110>70.5^{\circ}$ & $<100>36.9^{\circ}$ & $<100>36.9^{\circ}$ & $<100>43.6^{\circ}$ & $<100>16.3^{\circ}$ \\
\hline$\gamma_{\mathrm{GB}}\left(\mathrm{J} / \mathrm{m}^{2}\right)$ & 1.02 & 1.46 & 1.21 & 1.61 & 1.57 \\
\hline $\begin{array}{l}\mathrm{GB} \\
\text { motion }\end{array}$ & immobile & fast & moderate & fast & slow \\
\hline
\end{tabular}




\section{Figure captions:}

Fig. 1. Illustration of how the thermal gradient is created in the GB-containing system. (a) Typical simulation setup for creating a thermal gradient. (b) The temperature profile along $\mathrm{z}$ direction (same as the thermal gradient direction). $\mathrm{L}_{\mathrm{z}}$ represents the total length of the simulation system in the $\mathrm{z}$ direction.

Fig. 2. Method for tracking GB positions during their motion. (a) Direct visualization of GB positions. Only uranium atoms are shown and they are all in black color. (b) Distinction of different grains using orientation parameter analysis. Blue atoms belong to grain 1 and red atoms belong to grain 2 .

Fig. 3. Four representative snapshots of GB positions at different simulation times for the migration of $\Sigma 5$ tilt GB driven by a thermal gradient. Only uranium atoms are shown for clarity. The orientation parameter analysis is used to differentiate atoms into different grains, represented by red and blue colors respectively. The dashed lines in each snapshot represent the initial positions of the two GBs.

Fig. 4. Comparison of the time-evolution of the GB position (represented by the normalized number of atoms in grain 1) for five GBs under similar thermal gradients.

Fig. 5. Arrhenius plots of uranium self-diffusion along different directions for four GBs (except for $\Sigma 3$ twin) studied in this work. (a) $\mathrm{X}$ direction. (b) $\mathrm{Y}$ direction. (c) $\mathrm{Z}$ direction.

Fig. 6. Extracting GB velocity from MD simulations for the $\Sigma 5$ tilt GB. (a) The $2^{\text {nd }}$ order polynomial fitting of the number of uranium atoms in grain 1 with time. (b) The estimated GB migration velocity as a function of temperature.

Fig. 7. Comparison of GB mobility between MD and experiments at different temperatures. The different symbols of experimental data represent the different sources of data either measured or collected by Ainscough et al [41]. The calculated mobility from MD simulation is based on a $\Sigma 5$ tilt GB. Noting that for this calculation the entropy difference $\left(\triangle S=8 \times 10^{3} \mathrm{~J} / \mathrm{mol} / \mathrm{K}\right)$ specified in the book by Gottstein and Shvindlerman [5] is used for calculating the thermal gradient driving force.

Fig. 8. The enthalpy per $\mathrm{UO}_{2}$ molecule as a function of temperature during a heating-cooling process in MD simulation.

Fig. 9. Two standard methods for calculating GB mobilities in $\mathrm{UO}_{2}$. (a) Curvature-driven GB migration method. The circular grain 2 is embedded in the matrix 1. (b) GB random-walk method. The dashed line illustrates the GB position. 
Fig. 10. Comparison of GB mobilities calculated from different methods in MD simulations and measured from sintering experiments. (a) Comparison of GB mobilities calculated from three different methods (thermal gradient driven, curvature driven, and random walk). (b) Comparison of GB mobilities between modeling and experiments.

Fig. 11. Comparison of curvature driving force and thermal gradient driving force at different grain sizes. The dashed line indicates the typical grain size in LWR fuels.

Fig. 12. In-plane stress profiles of the $\Sigma 5$ tilt GB along the $\mathrm{z}$ direction before GBs start to migrate and after the two GBs anneal. The positive and negative signs indicate tensile and compressive stresses, respectively. 


\section{References}

[1] E. O. Hall, The Deformation and Ageing of Mild Steel: III Discussion of Results, Proceedings of the Physical Society of London Section B 64, 747 (1951).

[2] X.-M. Bai et al., Efficient Annealing of Radiation Damage Near Grain Boundaries via Interstitial Emission, Science 327, 1631 (2010).

[3] H.-S. Yang, G. R. Bai, L. J. Thompson, and J. A. Eastman, Interfacial thermal resistance in nanocrystalline yttria-stabilized zirconia, Acta Materialia 50, 2309 (2002).

[4] A. F. Mayadas, M. Shatzkes, and J. F. Janak, Electrical resistivity model for polycrystalline films: the case of specular reflection at external surfaces, Applied Physics Letters 14, 345 (1969).

[5] G. Gottstein, and L. S. Shvindlerman, Grain Boundary Migration in Metals: Thermodynamics, Kinetics, Applications (CRC Press, Boca Raton, Florida, 1999).

[6] R. Viswanathan, and C. L. Bauer, Kinetics of grain boundary migration in copper bicrystals with [001] rotation axes, Acta Metallurgica 21, 1099 (1973).

[7] J. W. Cahn, Y. Mishin, and A. Suzuki, Coupling grain boundary motion to shear deformation, Acta Materialia 54, 4953 (2006).

[8] B. Schönfelder, D. Wolf, S. R. Phillpot, and M. Furtkamp, Molecular-Dynamics Method for the Simulation of Grain-Boundary Migration, Interface Science 5, 245 (1997).

[9] M. Upmanyu, R. W. Smith, and D. J. Srolovitz, Atomistic Simulation of Curvature Driven Grain Boundary Migration, Interface Science 6, 41 (1998).

[10] Z. T. Trautt, M. Upmanyu, and A. Karma, Interface Mobility from Interface Random Walk, Science 314, 632 (2006).

[11] K. G. F. Janssens et al., Computing the mobility of grain boundaries, Nature Materials 5 , 124 (2006).

[12] J. K. Fink, Thermophysical properties of uranium dioxide, Journal of Nuclear Materials 279, 1 (2000).

[13] R. W. R. W. Powell, C. Y. Ho, and P. E. Liley, Thermal conductivity of selected materials (U. S. Dept. of Commerce, National Bureau of Standards, Washington, D. C., 1966).

[14] C. Ronchi, M. Sheindlin, D. Staicu, and M. Kinoshita, Effect of burn-up on the thermal conductivity of uranium dioxide up to $100.000 \mathrm{MWdt}$-1, Journal of Nuclear Materials 327, 58 (2004).

[15] D. R. Olander, Fundamental Aspects of Nuclear Reactor Fuel Elements (Technical Information Center, Office of Public Affairs, Energy Research and Development Administration, 1976).

[16] M. I. Mendelev, C. Deng, C. A. Schuh, and D. J. Srolovitz, Comparison of molecular dynamics simulation methods for the study of grain boundary migration, Modelling and Simulation in Materials Science and Engineering 21, 045017 (2013). 
[17] S. Plimpton, Fast Parallel Algorithms for Short-Range Molecular Dynamics, Journal of Computational Physics 117, 1 (1995).

[18] C. B. Basak, A. K. Sengupta, and H. S. Kamath, Classical molecular dynamics simulation of UO2 to predict thermophysical properties, Journal of Alloys and Compounds 360, 210 (2003).

[19] R. Devanathan, J. G. Yu, and W. J. Weber, Energetic recoils in UO2 simulated using five different potentials, Journal of Chemical Physics 130, 174502 (2009).

[20] S. A. Taller, and X.-M. Bai, Assessment of structures and stabilities of defect clusters and surface energies predicted by nine interatomic potentials for UO2, Journal of Nuclear Materials 443, 84 (2013).

[21] P. Nerikar et al., Intrinsic electrostatic effects in nanostructured ceramics, Physical Review B 81, 064111 (2010).

[22] X. M. Bai, A. El-Azab, J. G. Yu, and T. R. Allen, Migration mechanisms of oxygen interstitial clusters in UO2, Journal of Physics-Condensed Matter 25, 015003 (2013).

[23] A. Chernatynskiy, C. Flint, S. Sinnott, and S. Phillpot, Critical assessment of UO2 classical potentials for thermal conductivity calculations, Journal of Materials Science 47, 7693 (2012).

[24] Y. Zhang et al., Crack tip plasticity in single crystal UO2: Atomistic simulations, Journal of Nuclear Materials 430, 96 (2012).

[25] Y. Zhang et al., Molecular dynamics simulations of intergranular fracture in UO2 with nine empirical interatomic potentials, Journal of Nuclear Materials 452, 296 (2014).

[26] T. G. Desai, P. Millett, M. Tonks, and D. Wolf, Atomistic simulations of void migration under thermal gradient in UO2, Acta Materialia 58, 330 (2010).

[27] T. G. Desai, and B. P. Uberuaga, Stress-induced phase transformation in nanocrystalline UO2, Scripta Materialia 60, 878 (2009).

[28] B. A. Luty, M. E. Davis, I. G. Tironi, and W. F. Van Gunsteren, A Comparison of Particle-Particle, Particle-Mesh and Ewald Methods for Calculating Electrostatic Interactions in Periodic Molecular Systems, Molecular Simulation 14, 11 (1994).

[29] P. V. Nerikar et al., Grain Boundaries in Uranium Dioxide: Scanning Electron Microscopy Experiments and Atomistic Simulations, Journal of the American Ceramic Society 94, 1893 (2011).

[30] H. Ogawa, GBstudio: a builder software on periodic models of CSL boundaries for molecular simulation, Materials Transactions 47, 2706 (2006).

[31] R. G. Hoagland, and R. J. Kurtz, The relation between grain-boundary structure and sliding resistance, Philosophical Magazine A 82, 1073 (2002).

[32] K. Govers, S. Lemehov, M. Hou, and M. Verwerft, Comparison of interatomic potentials for UO2: Part II: Molecular dynamics simulations, Journal of Nuclear Materials 376, 66 (2008). 
[33] D. Manara et al., Melting of stoichiometric and hyperstoichiometric uranium dioxide, Journal of Nuclear Materials 342, 148 (2005).

[34] W. G. Hoover, Constant-pressure equations of motion, Physical Review A 34, 2499 (1986).

[35] S. Nose, A unified formulation of the constant temperature molecular dynamics methods, The Journal of Chemical Physics 81, 511 (1984).

[36] M. P. Allen, and D. J. Tildesley, Computer Simulation of Liquids (Oxford University Press, New York, 1989).

[37] P. Jund, and R. Jullien, Molecular-dynamics calculation of the thermal conductivity of vitreous silica, Physical Review B 59, 13707 (1999).

[38] H. Zhang, M. Upmanyu, and D. J. Srolovitz, Curvature driven grain boundary migration in aluminum: molecular dynamics simulations, Acta Materialia 53, 79 (2005).

[39] P.-L. Chen, and I. W. Chen, Role of Defect Interaction in Boundary Mobility and Cation Diffusivity of CeO2, Journal of the American Ceramic Society 77, 2289 (1994).

[40] B. Schönfelder, G. Gottstein, and L. S. Shvindlerman, Comparative study of grainboundary migration and grain-boundary self-diffusion of [001] twist-grain boundaries in copper by atomistic simulations, Acta Materialia 53, 1597 (2005).

[41] J. B. Ainscough, B. W. Oldfield, and J. O. Ware, Isothermal grain growth kinetics in sintered UO2 pellets, Journal of Nuclear Materials 49, 117 (1973).

[42] C. S. Smith, Grains, Phases, and Interfaces: an Interpretation of Microstructure, Trans. AIME 175, 15 (1948).

[43] M. I. Mendelev, D. J. Srolovitz, and W. E, Grain-boundary migration in the presence of diffusing impurities: Simulations and analytical models, Philosophical Magazine A 81, 2243 (2001).

[44] C. B. Carter, and S. L. Sass, Electron Diffraction and Microscopy Techniques for Studying Grain-Boundary Structure, Journal of the American Ceramic Society 64, 335 (1981).

[45] X. M. Bai, and M. Li, Differences between solid superheating and liquid supercooling, Journal of Chemical Physics 123 (2005).

[46] D. A. Porter, and K. E. Easterling, Phase Transformations in Metals and Alloys (Chapman \& Hall, London, 1992).

[47] M. R. Tonks et al., Guidance to design grain boundary mobility experiments with molecular dynamics and phase-field modeling, Acta Materialia 61, 1373 (2013).

[48] A. Karma, Z. T. Trautt, and Y. Mishin, Relationship between Equilibrium Fluctuations and Shear-Coupled Motion of Grain Boundaries, Physical Review Letters 109, 095501 (2012).

[49] Z. T. Trautt, and Y. Mishin, Grain boundary migration and grain rotation studied by molecular dynamics, Acta Materialia 60, 2407 (2012).

[50] C. Deng, and C. A. Schuh, Atomistic Simulation of Slow Grain Boundary Motion, Physical Review Letters 106, 045503 (2011). 
[51] M. R. Tonks, Y. Zhang, X. Bai, and P. C. Millett, Demonstrating the Temperature Gradient Impact on Grain Growth in UO2 Using the Phase Field Method, Materials Research Letters 2, 23 (2014).

[52] M. Tonks, P. Millett, W. Cai, and D. Wolf, Analysis of the elastic strain energy driving force for grain boundary migration using phase field simulation, Scripta Materialia 63, 1049 (2010). 


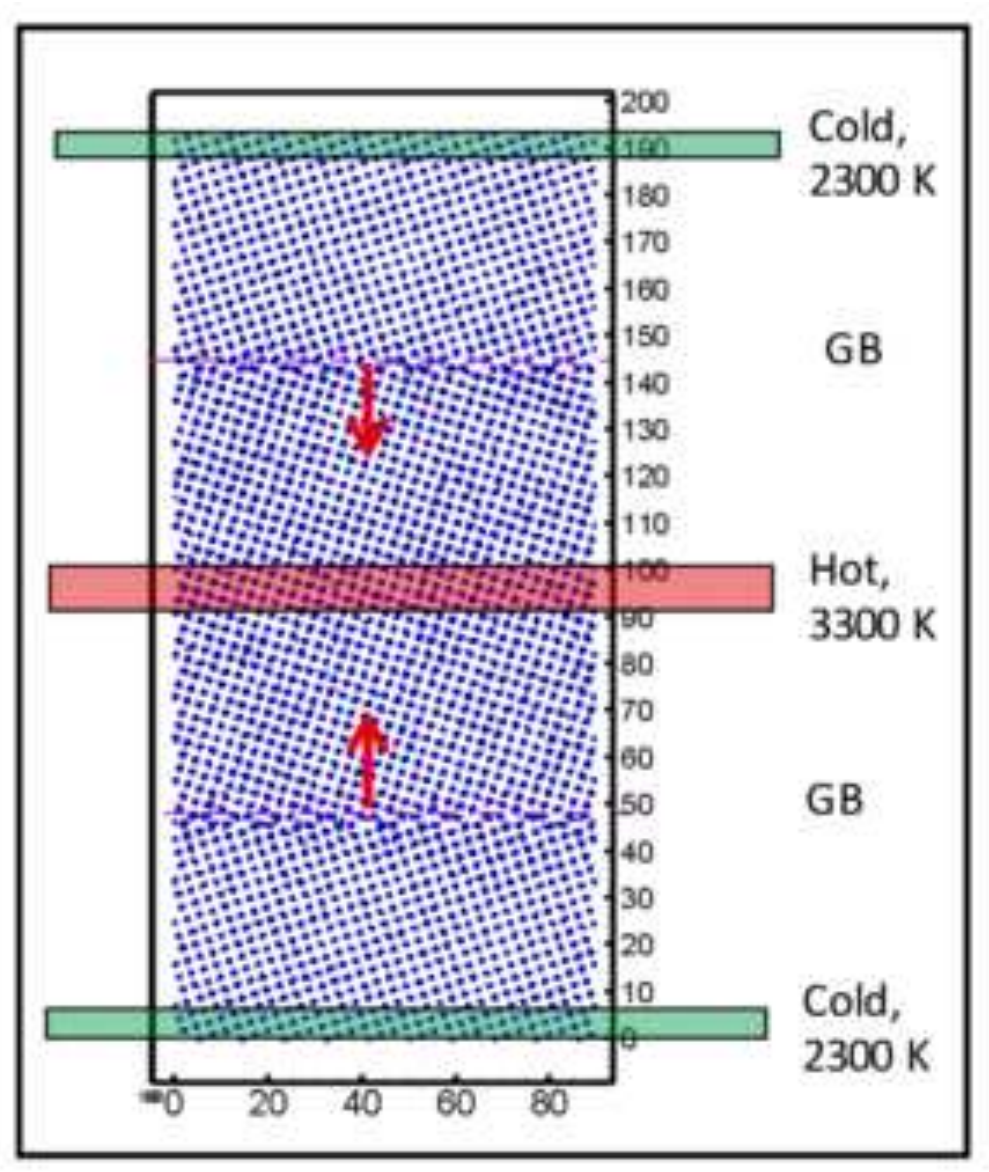

(a)

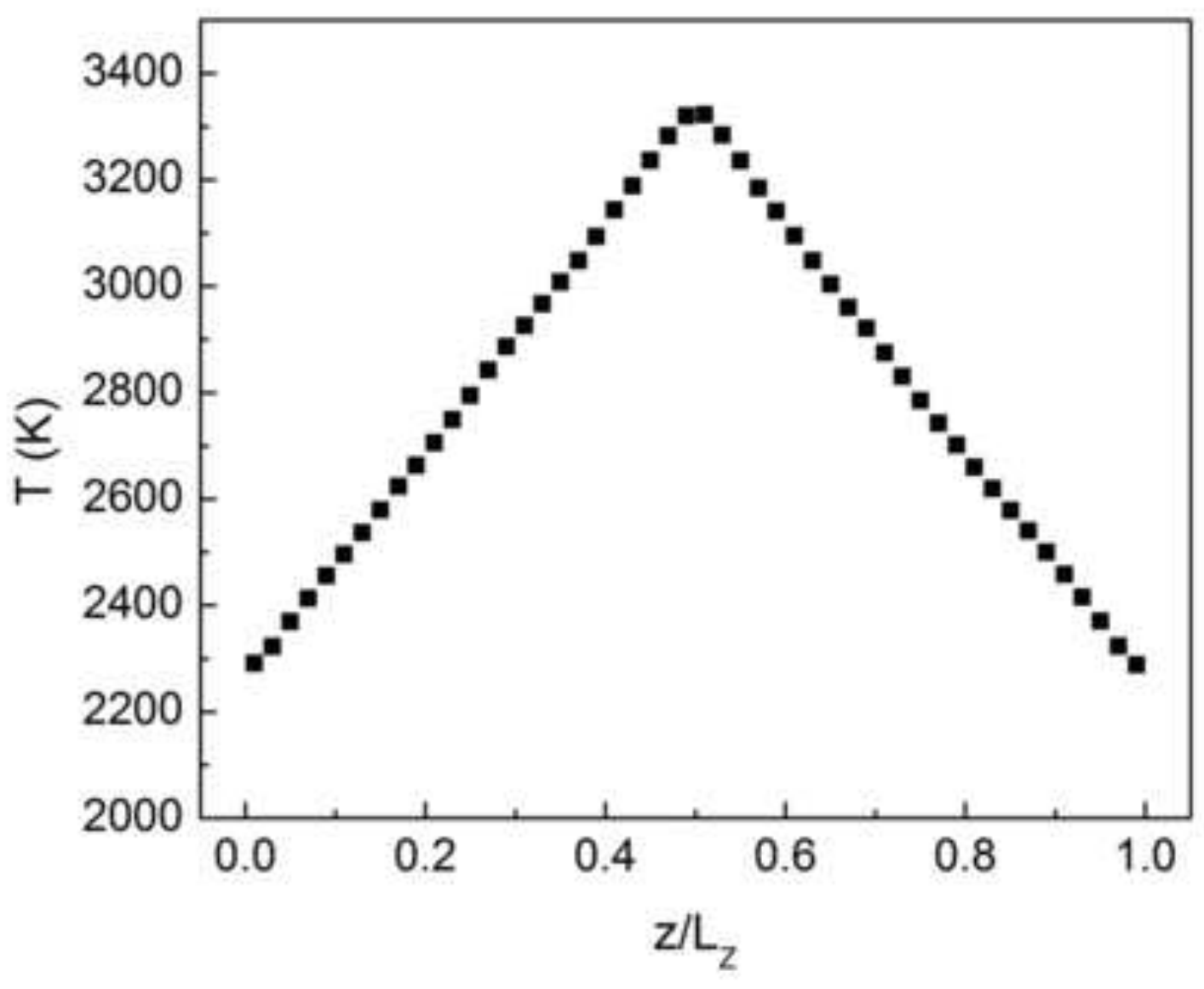

(b) 


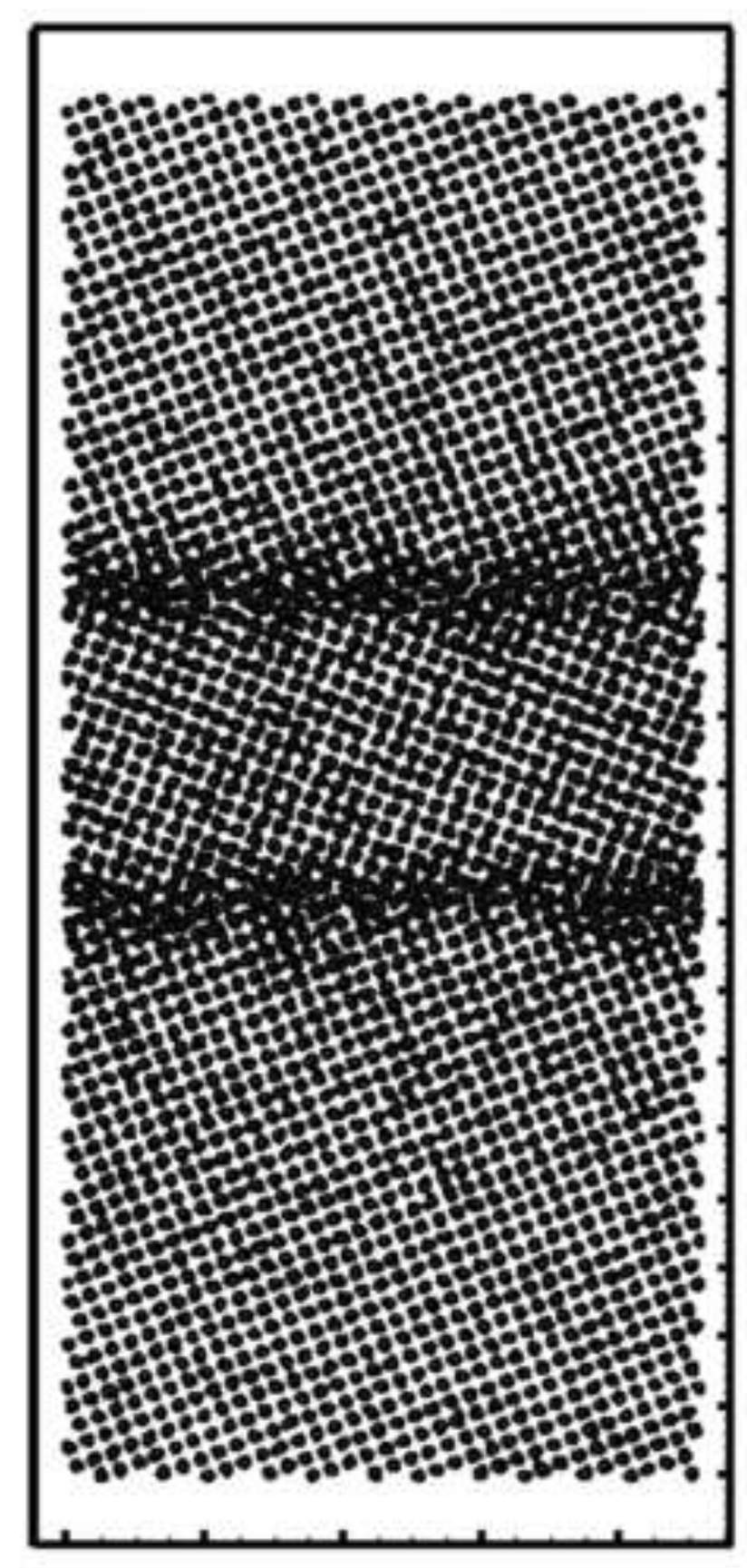

(a)

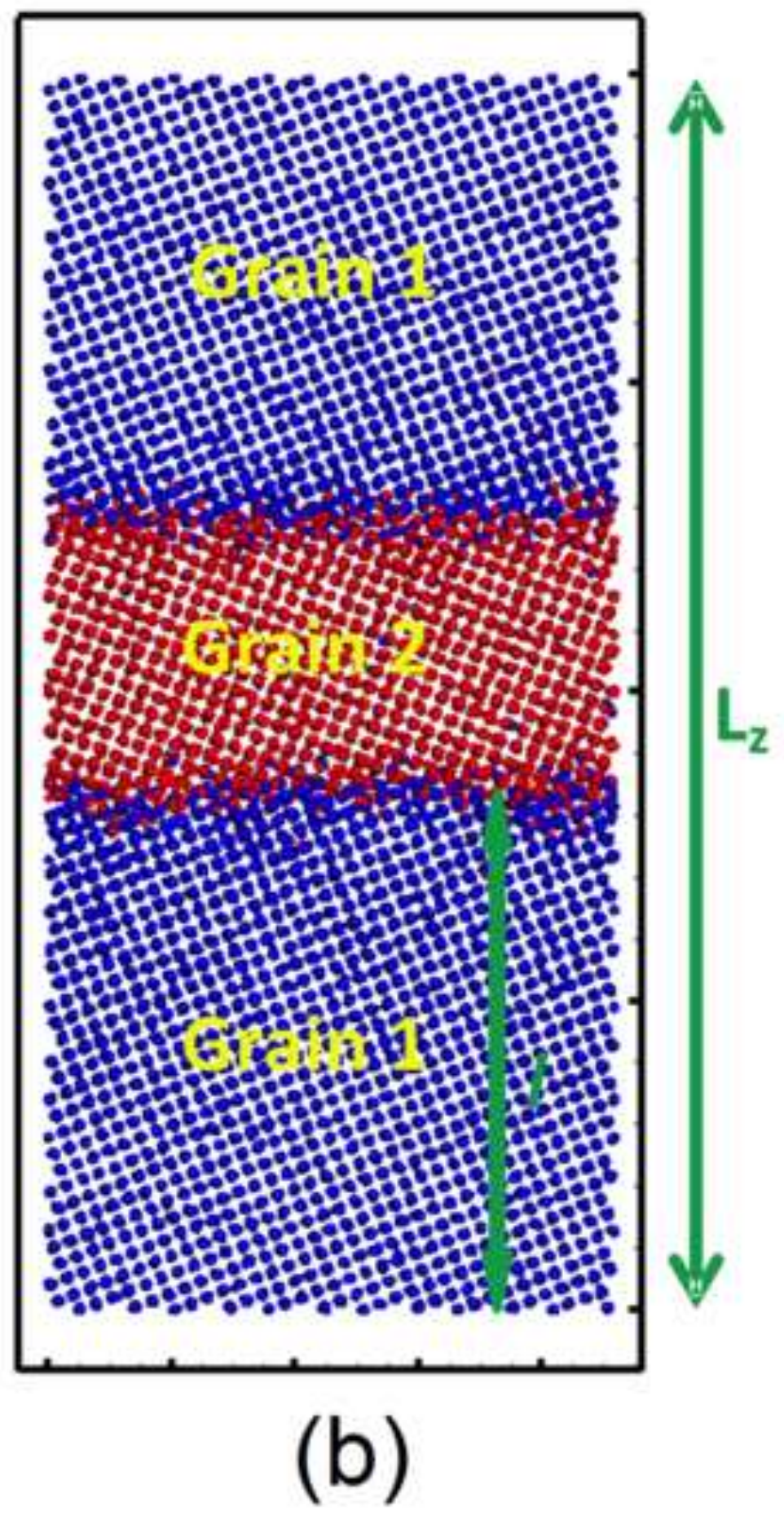




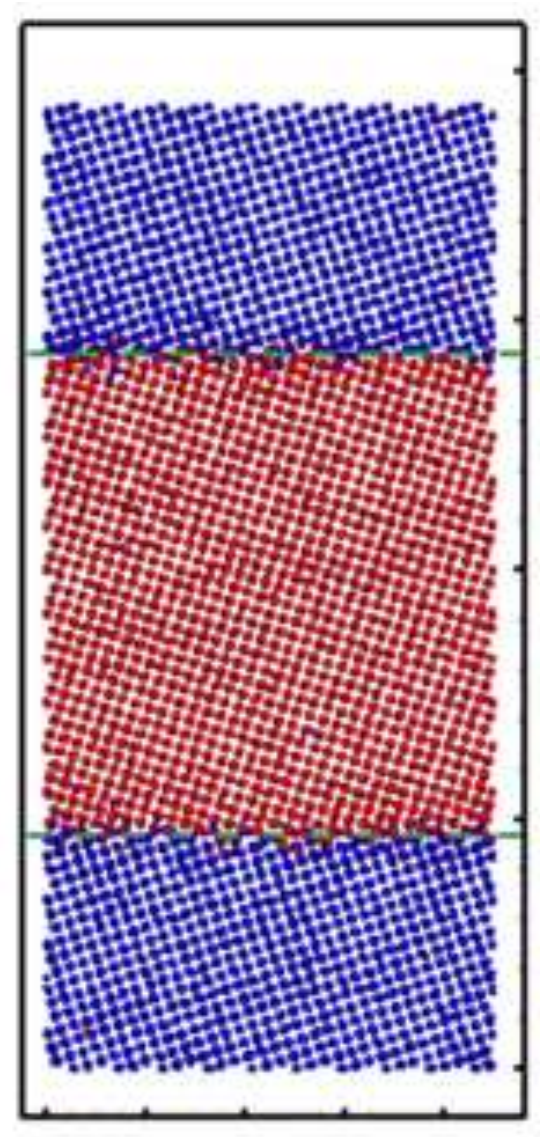

(a) $\mathrm{t}=0.0 \mathrm{~ns}$

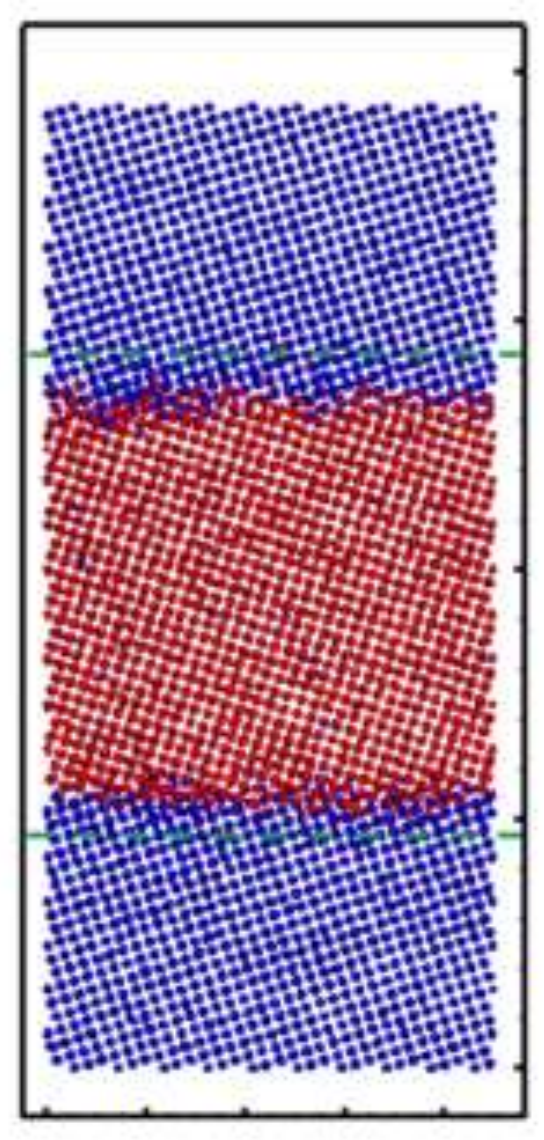

(b) t $=0.8 \mathrm{~ns}$

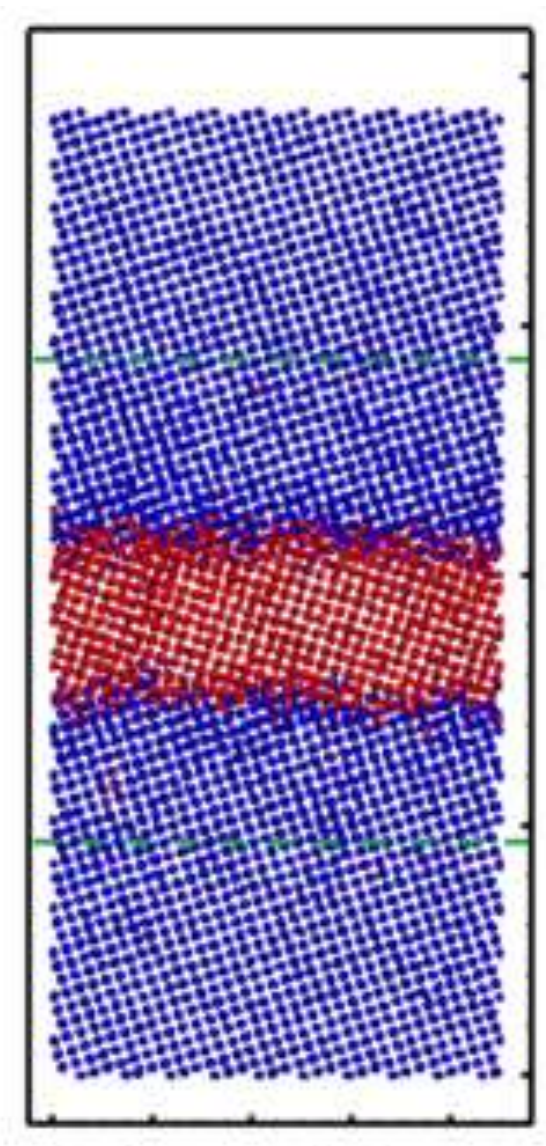

(c) $t=1.6 \mathrm{~ns}$

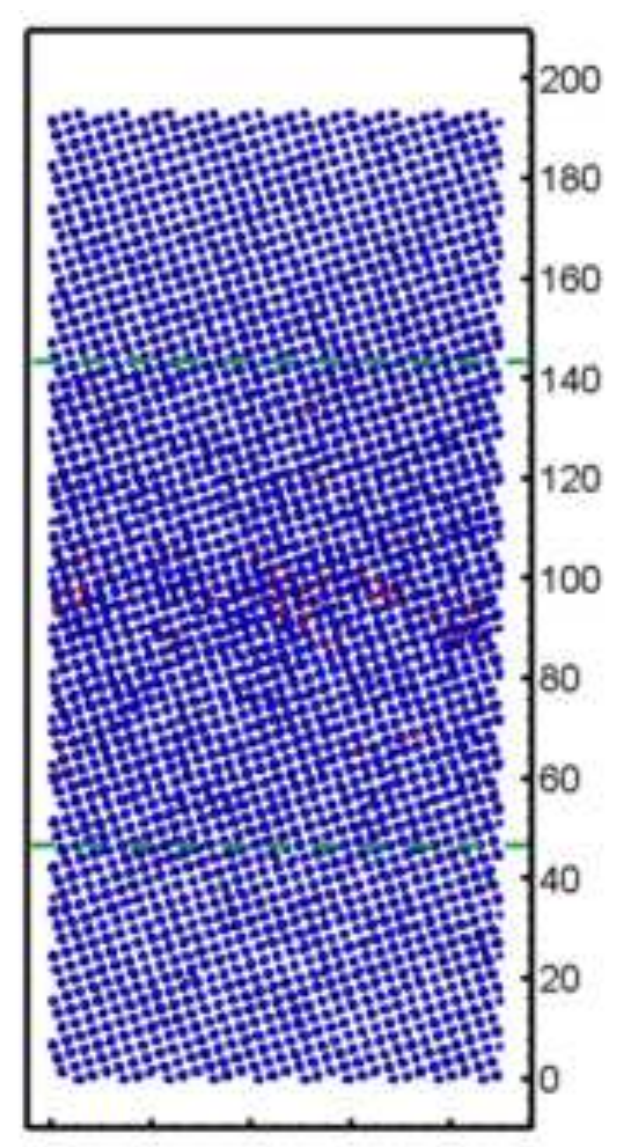

(d) $\mathrm{t}=1.8 \mathrm{~ns}$ 


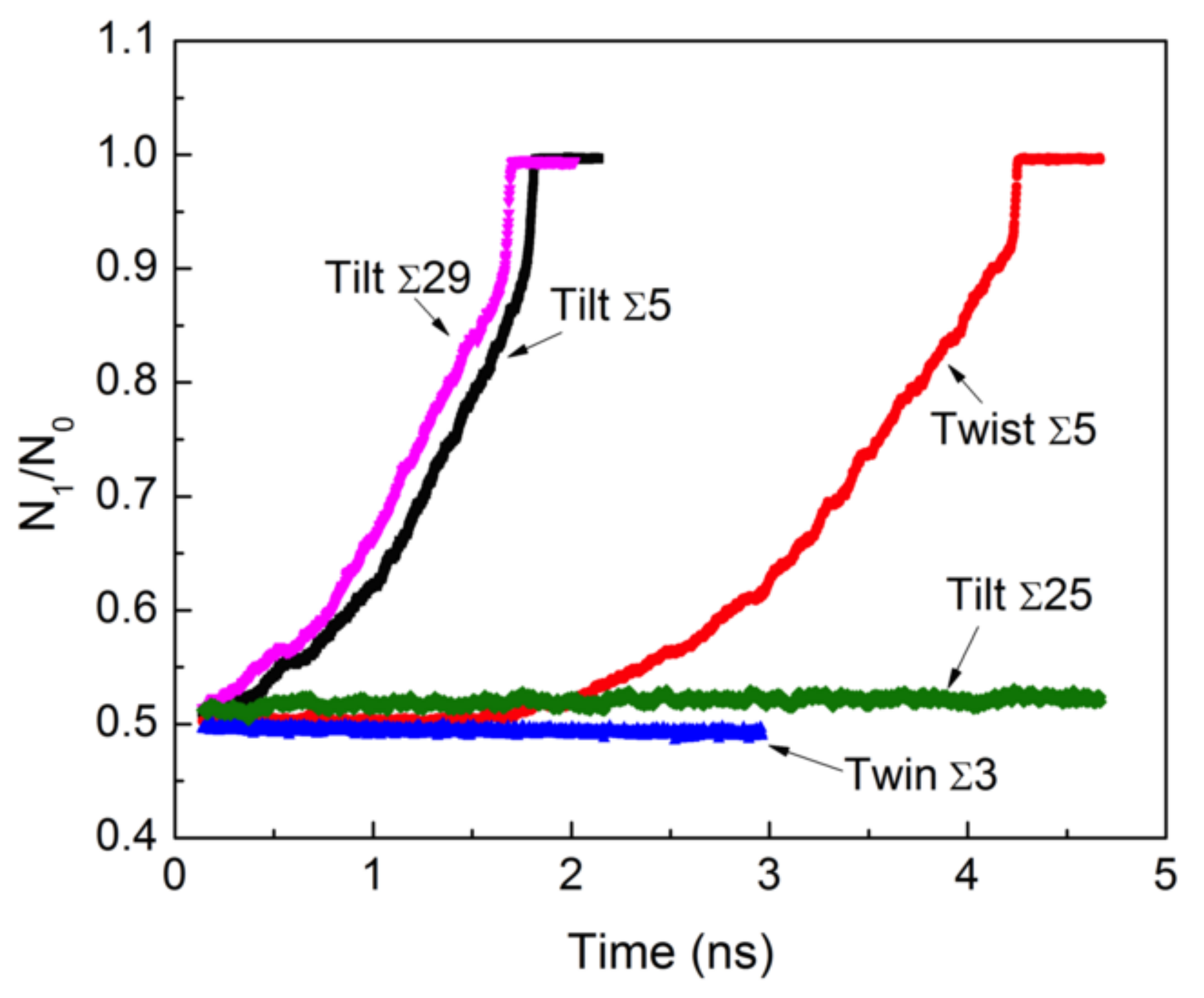

5

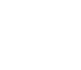

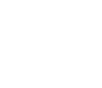



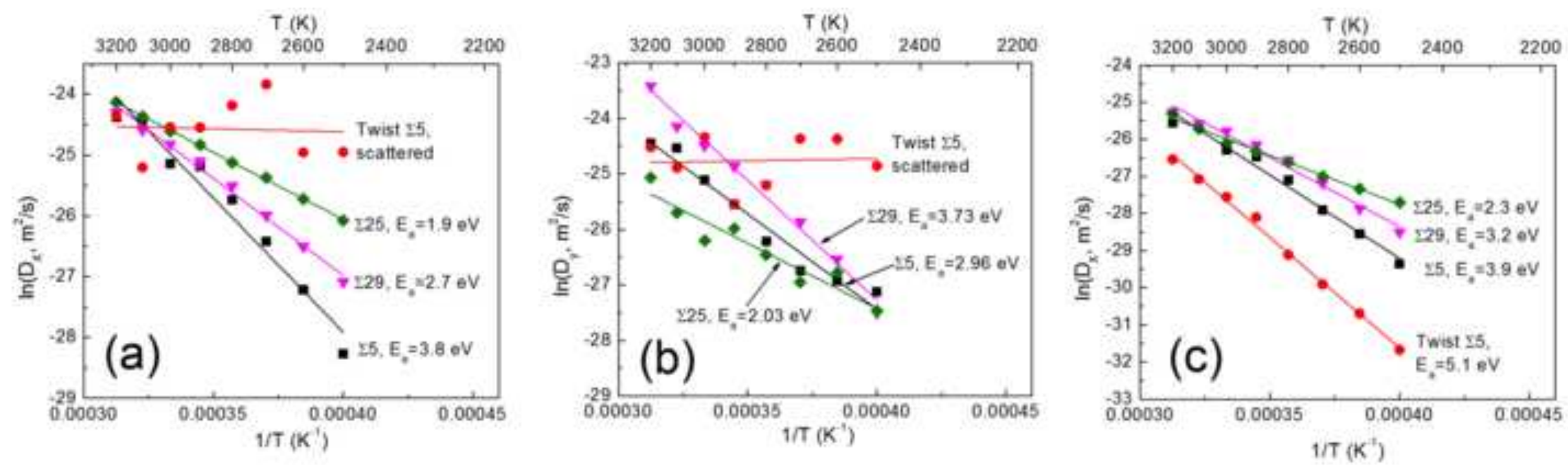

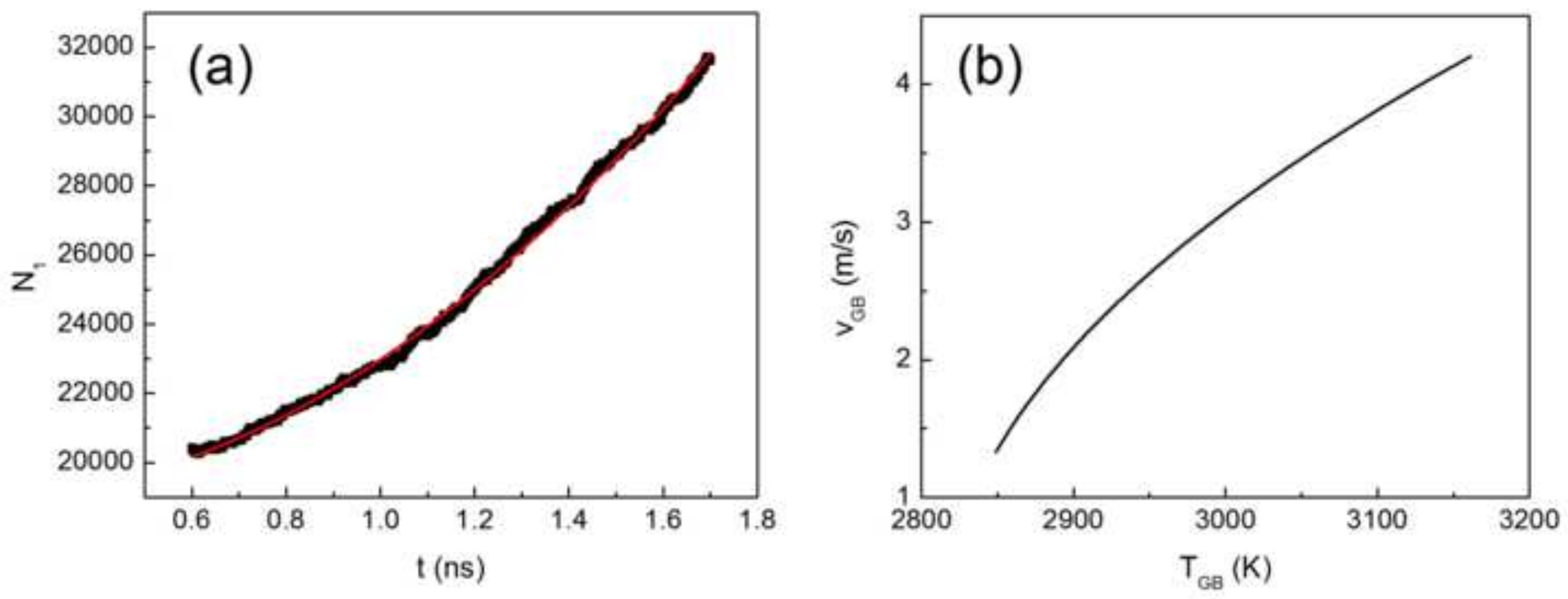


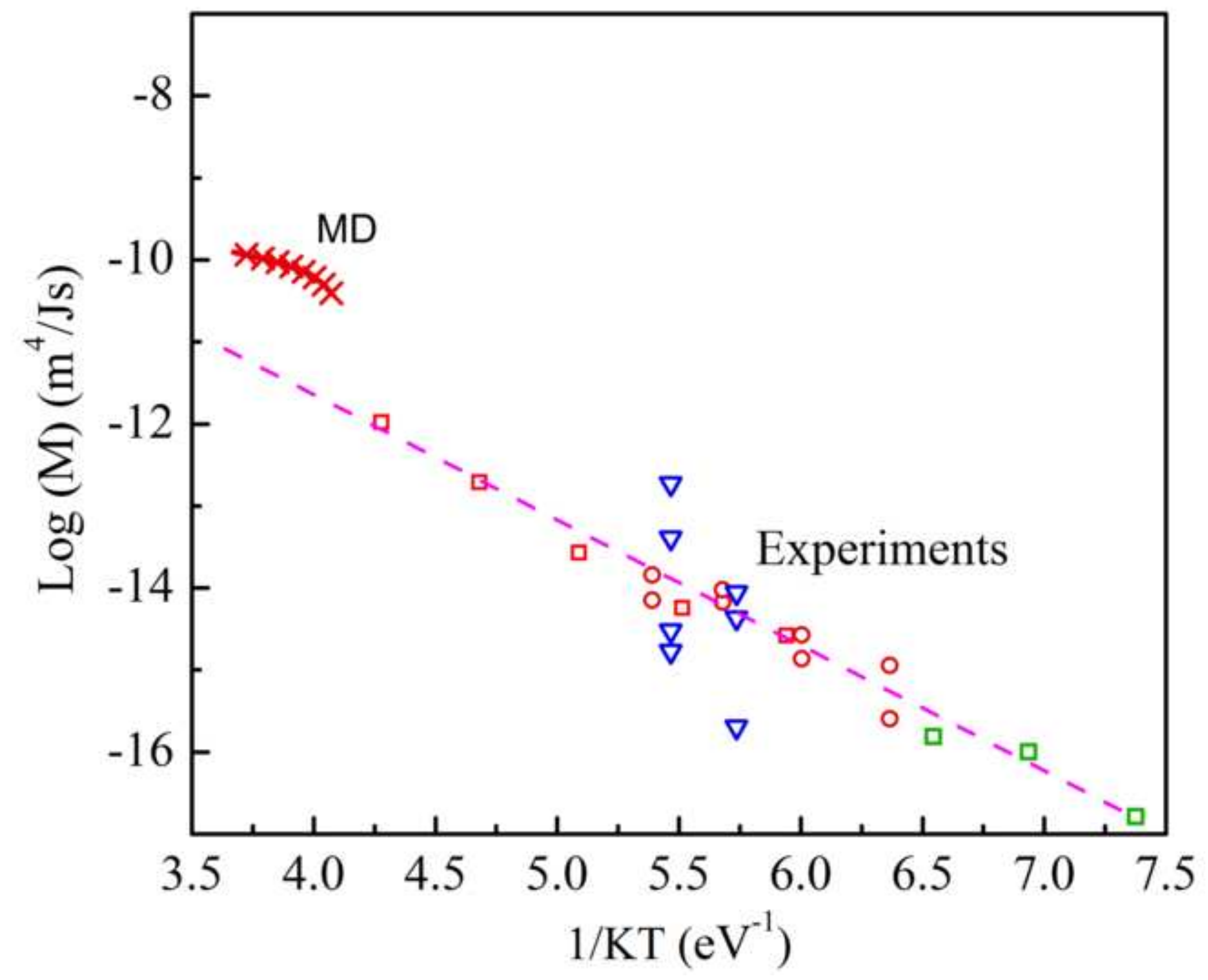




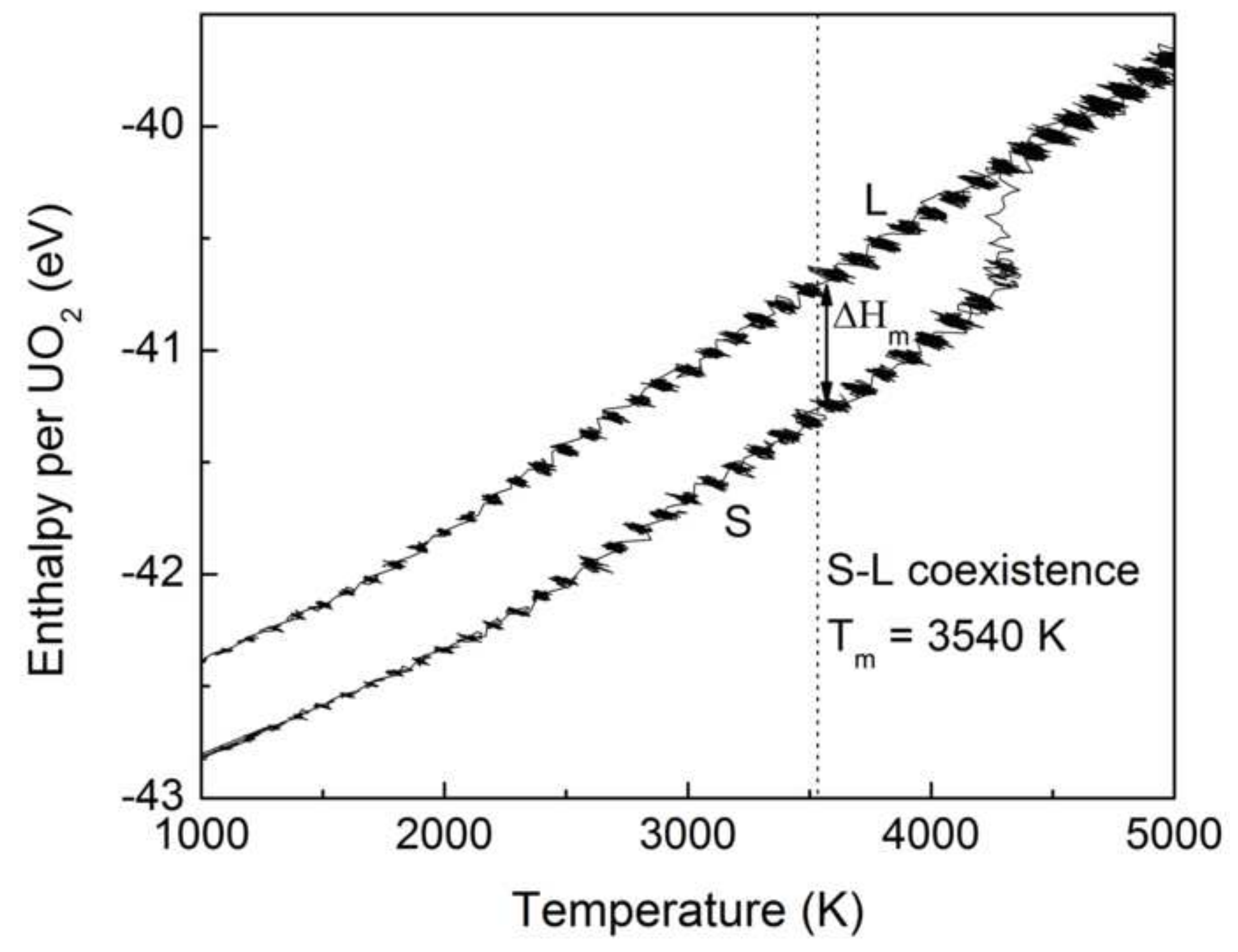



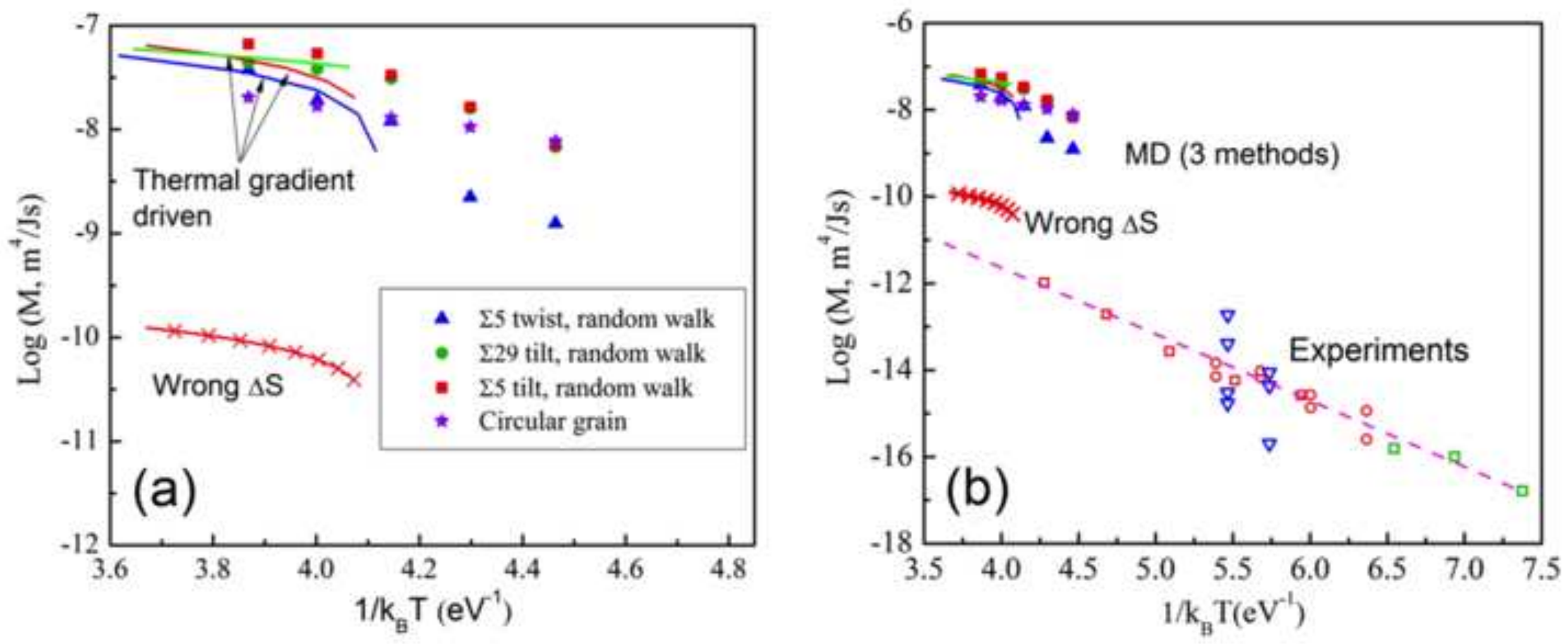


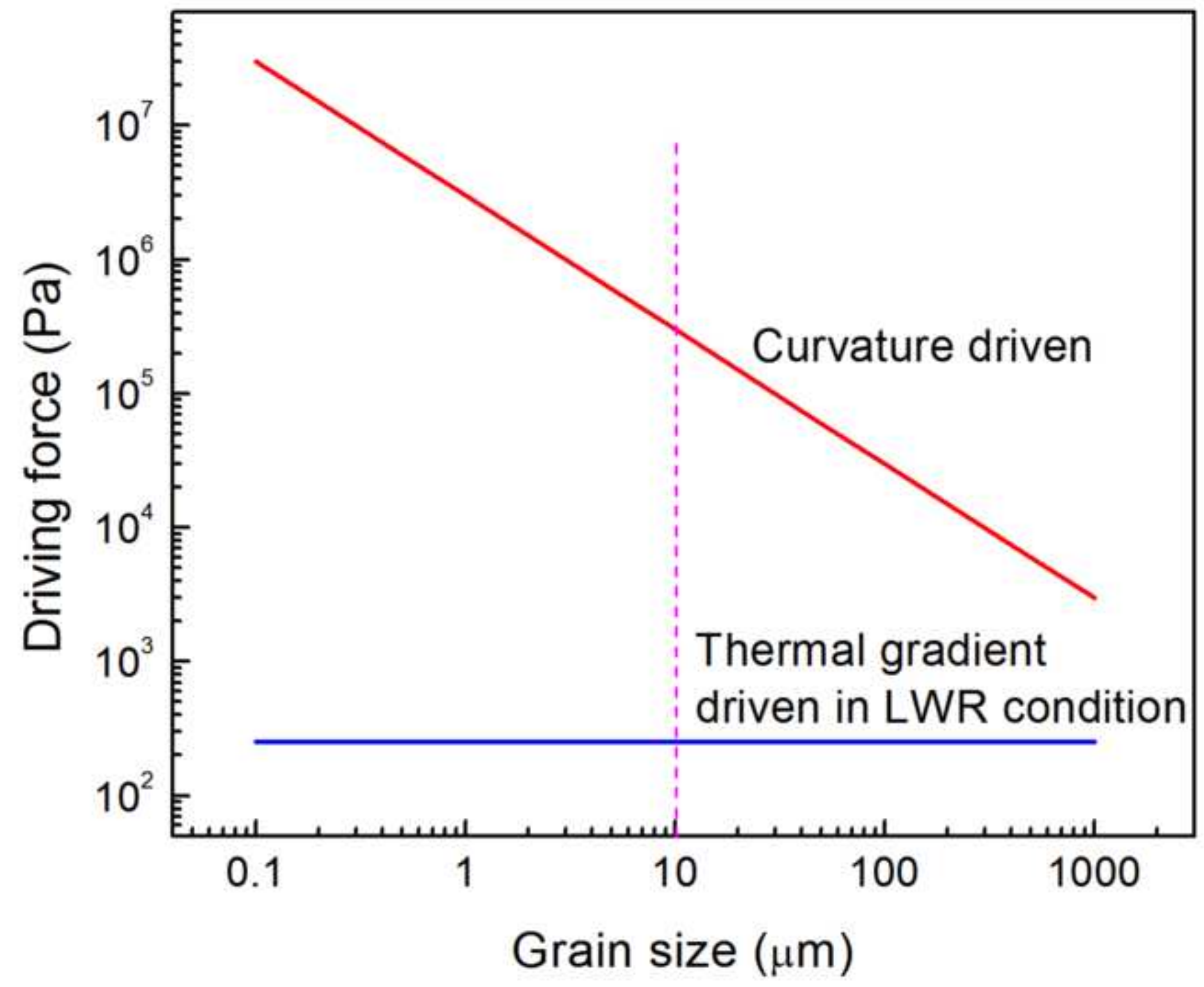




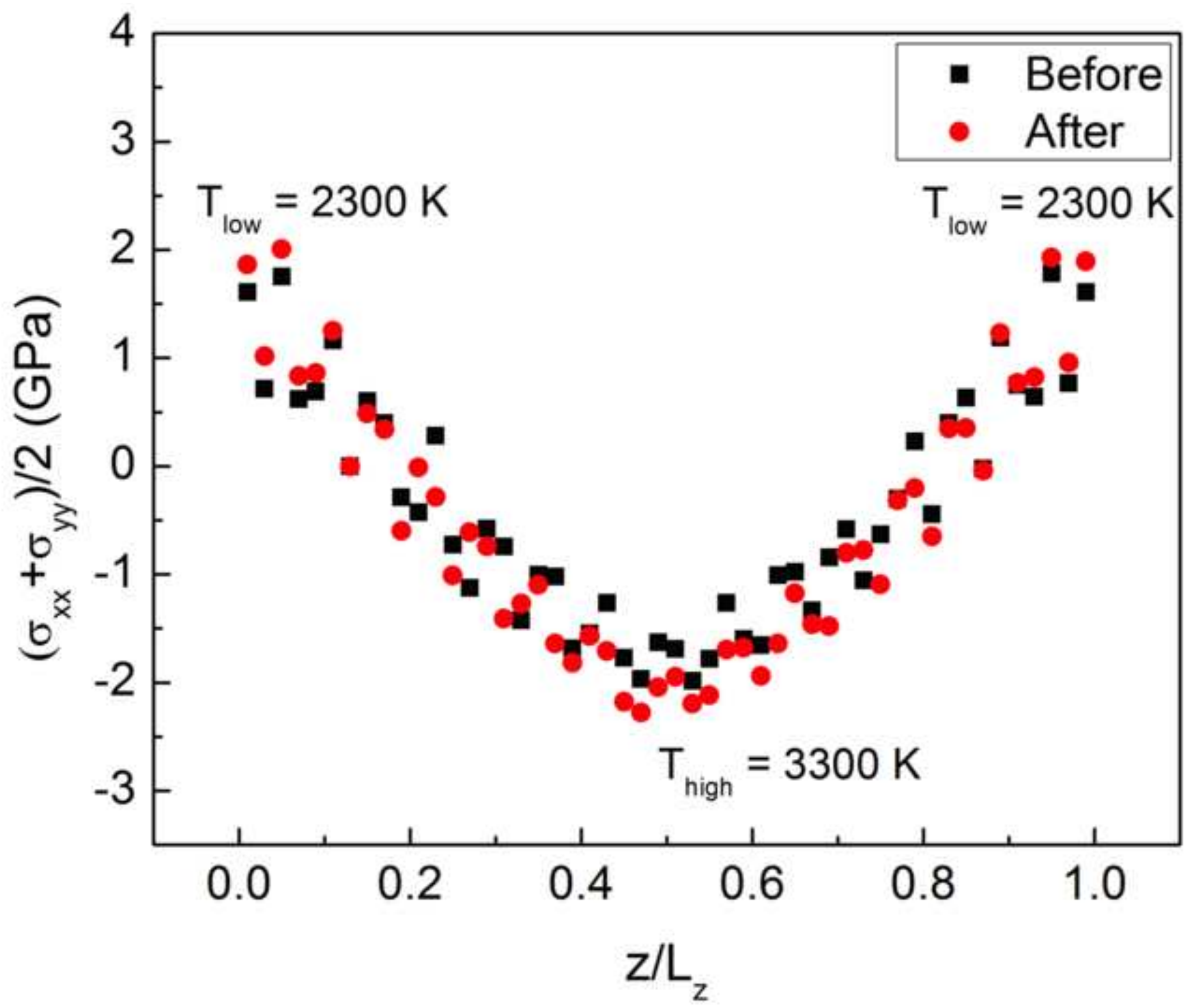

